\title{
Representações da Equação de Dirac em 1+1 Dimensões
}

\section{Representations of the Dirac Equation in 1+1 Dimensions}

\author{
Andrei Smirnov*, Antonio Jorge Dantas Farias Jr. \\ Departamento de Física, Universidade Federal de Sergipe, Sergipe, Brasil.
}

\begin{abstract}
Recebido em 11 de março de 2016. Revisado em 5 de abril de 2016. Aceito em 11 de abril de 2016
Neste trabalho descrevemos uma classificação geral das representações da equação de Dirac em $1+1$ dimensões. Na classificação são incluídas as representações nas quais cada matriz de Dirac $\beta, \alpha$ é associada a uma única matriz de Pauli. Esta classificação inclui 6 representações principais. A inclusão das representações com sinais modificados das matrizes de Pauli aumenta o número de representações distintas até 24. Determinamos as transformações unitárias entre todas as representações na forma explícita. O estudo da estrutura do conjunto das transformações leva à conclusão de que todas as representações são equivalentes, isto é, uma representação pode ser obtida a partir de qualquer outra por uma transformação unitária. Estabelecemos que o conjunto das transformações forma um grupo não abeliano com respeito ao produto matricial em classes de equivalência, definidas através da indistinguibilidade das transformações que diferem por um fator de fase. Este grupo possui um subgrupo não trivial, o qual por sua vez contém dois subgrupos não triviais. Apresentamos a forma geral das matrizes de Dirac em $1+1$ dimensões determinada por 3 parâmetros arbitrários. Demonstramos a existência de isomorfismo entre o par das matrizes de Dirac e o par de vetores unitários ortogonais. Apresentamos também aplicações dos resultados obtidos neste trabalho.
\end{abstract}

Palavras-chave: equação de Dirac, $1+1$ dimensões, transformações unitárias, grupos finitos.

In this paper a general classification of representations of the Dirac equation in $1+1$ dimensions is described. There are considered such representations in which every Dirac matrix $\beta, \alpha$ is associated with a unique Pauli matrix. The classification includes 24 representations among which six representations are main and others are ones with sign modified Pauli matrices. The unitary transformations between all the representations are determined in the explicit form. The study of structure of the set of transformations results in conclusions that all the representations are equivalent, so that anyone of the representations can be obtained from any other by a unitary transformation. It is established that the set of the transformations forms a non-abelian group in respect of matrix product in equivalence classes, which are defined through indistinguishability of transformations, which differ by a phase factor. The group possesses one non-trivial subgroup, which in its turn includes two non-trivial subgroups. The most general form of the Dirac matrices in $1+1$ dimensions determined by three arbitrary parameters is presented. Isomorphism of the couple of the Dirac matrices to the couple of orthogonal unitary vectors is demonstrated. Applications of the results obtained in the paper are presented.

Keywords: Dirac equation, $1+1$ dimensions, unitary transformations, finite groups.

\section{Introdução}

Neste artigo discutimos algumas questões da teoria de Dirac em 1+1 dimensões. Antes de começar esta discussão, relembramos alguns aspectos da teoria de Dirac no caso usual de $3+1$ dimensões, que

\footnotetext{
*Endereço de correspondência: smirnov.globe@gmail.com.
}

são mais relevantes ao nosso trabalho. Mencionamos que existe vasto material dedicado à equação de Dirac, tanto nos livros de texto de Mecânica Quântica e de Teoria de Campos, como se comprova nas Refs. [1], [2], [3] e nas Refs. [4], [5], [6], [7], respec- 
tivamente; quanto em livros especializados, também compovado nas Refs. [8], [9].

Primeiramente, apresentamos uma dedução formal da equação de Dirac para os leitores não familiarizados com esta área. Iniciamos pela lei de conservação de energia da mecânica: a energia total de um sistema mecânico conservativo é constante. Para uma partícula temos:

$$
K+V=E=\text { const }
$$

onde $K$ é a energia cinética, $V$ é a energia potencial e $E$ é a energia total da partícula. Expressamos a energia cinética em termos do momento da partícula $\vec{p}$ :

$$
K=\frac{\vec{p}^{2}}{2 m}
$$

onde $m$ é a massa da partícula. Observamos que a energia potencial é uma função das coordenadas $V=V(\vec{r})$. Escrevemos a Eq. (1) na forma:

$$
E=\frac{\vec{p}^{2}}{2 m}+V(\vec{r}) \text {. }
$$

Para construir a equação de onda não relativística, a partir da Eq. (2), fazemos uma substituição da energia total, das coordenadas e dos momentos por seus operadores correspondentes que atuam em uma função de onda $\psi$ :

$$
\begin{aligned}
& E \rightarrow \hat{E} \psi=i \hbar \frac{\partial \psi}{\partial t}, x \rightarrow \hat{x} \psi=x \psi, \\
& y \rightarrow \hat{y}=y \psi, z \rightarrow \hat{z}=z \psi, \\
& p_{x} \rightarrow \hat{p}_{x} \psi=-i \hbar \frac{\partial \psi}{\partial x}, p_{y} \rightarrow \hat{p}_{y} \psi=-i \hbar \frac{\partial \psi}{\partial y}, \\
& p_{z} \rightarrow \hat{p}_{z} \psi=-i \hbar \frac{\partial \psi}{\partial z} .
\end{aligned}
$$

Substituindo os operadores da Eq. (3) na Eq. (2) chegamos a equação de onda:

$i \hbar \frac{\partial \psi}{\partial t}=-\frac{\hbar^{2}}{2 m}\left(\frac{\partial^{2} \psi}{\partial x^{2}}+\frac{\partial^{2} \psi}{\partial y^{2}}+\frac{\partial^{2} \psi}{\partial z^{2}}\right)+V(x, y, z) \psi$.

A equação (4) é a equação de Schrödinger, que descreve uma partícula quântica não relativística sujeita a um campo potencial $V$. A derivada em relação ao tempo de primeira ordem no lado esquerdo da
Eq. (4) possibilita descrever a evolução temporal da partícula. Na construção da equação de onda de uma partícula relativística é necessário obter uma equação de onda em que a derivada em relação ao tempo é também de primeira ordem.

Para uma partícula relativística, temos a seguinte fórmula da energia relativística $E$ :

$$
\begin{aligned}
& E^{2}=\vec{p}^{2} c^{2}+\left(m c^{2}\right)^{2}=p_{x}^{2} c^{2}+p_{y}^{2} c^{2}+p_{z}^{2} c^{2} \\
& +\left(m c^{2}\right)^{2}
\end{aligned}
$$

onde $m$ é a massa da partícula, $c$ é a velocidade de luz e $\vec{p}$ é o momento relativístico. Fazendo uma substituição das quantidades na Eq. (5) pelos operadores da Eq. (3), obtemos a equação:

$-\hbar^{2} \frac{\partial^{2} \psi}{\partial t^{2}}=-c^{2} \hbar^{2} \frac{\partial^{2} \psi}{\partial x^{2}}-c^{2} \hbar^{2} \frac{\partial^{2} \psi}{\partial y^{2}}-c^{2} \hbar^{2} \frac{\partial^{2} \psi}{\partial z^{2}}+\left(m c^{2}\right)^{2}$,

dividindo-a por $c^{2} \hbar^{2}$ temos

$$
\frac{1}{c^{2}} \frac{\partial^{2} \psi}{\partial t^{2}}-\frac{\partial^{2} \psi}{\partial x^{2}}-\frac{\partial^{2} \psi}{\partial y^{2}}-\frac{\partial^{2} \psi}{\partial z^{2}}+\left(\frac{m c}{\hbar}\right)^{2}=0 .
$$

Na Eq. (6) a derivada em relação ao tempo é de segunda ordem devido ao quadrado da energia na Eq. (5). Aplicando a raiz quadrada na Eq. (5), obtemos:

$$
\begin{array}{r}
E=\sqrt{\vec{p}^{2} c^{2}+\left(m c^{2}\right)^{2}}= \\
\sqrt{p_{x}^{2} c^{2}+p_{y}^{2} c^{2}+p_{z}^{2} c^{2}+\left(m c^{2}\right)^{2}},
\end{array}
$$

onde o termo da energia é de primeira ordem. Entretanto, a raiz quadrada no lado direito dificulta a interpretação deste termo depois da substituição das componentes do momento pelos operadores. A análise dimensional das quantidades do lado direito da Eq. (7) demonstra que os termos $p_{x} c, p_{y} c, p_{z} c$ e $m c^{2}$ podem ser escritos na forma de uma combinação linear com alguns coeficientes $\alpha_{1}, \alpha_{2}, \alpha_{3} \mathrm{e}$ $\beta$ :

$$
E=\alpha_{1} p_{x} c+\alpha_{2} p_{y} c+\alpha_{3} p_{z} c+\beta m c^{2} .
$$

Elevando ambos os lados da Eq. (8) ao quadrado e mantendo a ordem de multiplicação dos coeficientes, temos:

$$
\begin{aligned}
E^{2}= & \left(\alpha_{1} p_{x} c+\alpha_{2} p_{y} c+\alpha_{3} p_{z} c+\beta m c^{2}\right) \\
& \times\left(\alpha_{1} p_{x} c+\alpha_{2} p_{y} c+\alpha_{3} p_{z} c+\beta m c^{2}\right)
\end{aligned}
$$




$$
\begin{aligned}
& =\left(\alpha_{1} \alpha_{2}+\alpha_{2} \alpha_{1}\right) p_{x} p_{y} c^{2}+\left(\alpha_{2} \alpha_{3}+\alpha_{3} \alpha_{2}\right) p_{y} p_{z} c^{2}+\left(\alpha_{3} \alpha_{1}+\alpha_{1} \alpha_{3}\right) p_{z} p_{x} c^{2} \\
& +\left(\alpha_{1} \beta+\beta \alpha_{1}\right) p_{x} c m c^{2}+\left(\alpha_{2} \beta+\beta \alpha_{2}\right) p_{y} c m c^{2}+\left(\alpha_{3} \beta+\beta \alpha_{3}\right) p_{z} c m c^{2} \\
& +\alpha_{1}^{2} p_{x}^{2} c^{2}+\alpha_{2}^{2} p_{y}^{2} c^{2}+\alpha_{3}^{2} p_{z}^{2} c^{2}+\beta^{2}\left(m c^{2}\right)^{2}
\end{aligned}
$$

Comparando a Eq. (9) com a Eq. (5), obtemos as seguintes condições para os coeficientes:

$$
\begin{aligned}
& \alpha_{1}^{2}=1, \alpha_{2}^{2}=1, \alpha_{3}^{2}=1, \beta^{2}=1, \\
& \alpha_{1} \alpha_{2}+\alpha_{2} \alpha_{1}=0, \alpha_{2} \alpha_{3}+\alpha_{3} \alpha_{2}=0, \alpha_{3} \alpha_{1}+\alpha_{1} \alpha_{3}=0, \\
& \alpha_{1} \beta+\beta \alpha_{1}=0, \alpha_{2} \beta+\beta \alpha_{2}=0, \alpha_{3} \beta+\beta \alpha_{3}=0 .
\end{aligned}
$$

Das condições da Eq. (10), conclui-se que os coeficientes $\alpha_{1}, \alpha_{2}, \alpha_{3}$ e $\beta$ não podem ser números, pois os múmeros obedecem à lei de comutação com respeito à multiplicação, isto é, para dois números $x_{1}$ e $x_{2}$, diferentes de zero, temos $x_{1} x_{2}=x_{2} x_{1}$ e $x_{1} x_{2}+x_{2} x_{1}=2 x_{1} x_{2}$. Considerando os coeficientes $\alpha_{1}, \alpha_{2}, \alpha_{3}$ e $\beta$ como matrizes, é possivel encontrar 4 matrizes que obedecem às condições da Eq. (10). A dimensão mínima destas matrizes é $4 \times 4$. As matrizes $\alpha_{1}, \alpha_{2}, \alpha_{3}$ e $\beta$ são chamadas de matrizes de Dirac.

Efetuando a substituição das quantidades na Eq. (8) pelos operadores da Eq. (3), chegamos a equação:

$$
\begin{aligned}
i \hbar \frac{\partial \psi}{\partial t}=- & \alpha_{1} i c \hbar \frac{\partial \psi}{\partial x}-\alpha_{2} i c \hbar \frac{\partial \psi}{\partial y} \\
& -\alpha_{3} i c \hbar \frac{\partial \psi}{\partial z}+\beta m c^{2} \psi
\end{aligned}
$$

que representa a equação de Dirac. A equação de Dirac descreve o comportamento quântico de uma partícula relativística de spin $1 / 2$, como por exemplo o elétron. Como a equação (11) é uma equação matricial, a função de onda $\psi$ é um objeto de 4 componentes, chamado de spinor de Dirac, que pode ser escrito na forma de uma coluna:

$$
\psi=\left(\begin{array}{l}
\psi_{1} \\
\psi_{2} \\
\psi_{3} \\
\psi_{4}
\end{array}\right) .
$$

Dividindo os lados esquerdo e direito da Eq. (11) por $c h$, obtemos:

$$
i \frac{1}{c} \frac{\partial \psi}{\partial t}=-\alpha_{1} i \frac{\partial \psi}{\partial x}-\alpha_{2} i \frac{\partial \psi}{\partial y}-\alpha_{3} i \frac{\partial \psi}{\partial z}+\beta \frac{m c}{\hbar} \psi
$$

Introduzimos as notações que são usadas na área da teoria relativística e de campos. As coordenadas espaciais e de tempo são reunidas em um quadrivetor $x^{\mu}$, em que o índice $\mu$ recebe os valores $0,1,2,3$ :

$$
\begin{aligned}
& x^{\mu}=\left(x^{0}, x^{1}, x^{2}, x^{3}\right) \\
& =\left(x^{0}, x^{i}\right)=\left(x^{0}, \vec{x}\right), i=1,2,3,
\end{aligned}
$$

com componentes

$$
x^{0}=c t, x^{1}=x, x^{2}=y, x^{3}=z .
$$

O 4-vetor $x^{\mu}$ representa os pontos do espaço-tempo. Para denotar as derivadas pelas coordenadas usamos o símbolo $\partial_{\mu}=\partial / \partial x^{\mu}$. Usaremos também as unidades naturais em que é aceita a convenção $c=\hbar=1$. Aplicando as notações e convenções indicadas acima na Eq. (13), temos:

$$
i \partial_{0} \psi=\left(-\alpha_{1} i \partial_{1}-\alpha_{2} i \partial_{2}-\alpha_{3} i \partial_{3}+\beta m\right) \psi \text {. }
$$

$\mathrm{Na}$ área da teoria relativística é usada também uma convenção de somatório por índices repetidos. Se em uma fórmula existe um termo de produto de quantidades que possuem o mesmo índice, isto significa que por este índice é efetuado um somatório. Neste caso o símbolo de soma não se escreve. Aplicamos esta convenção para o somatório dos termos semelhantes do lado direito da Eq. 15.

$$
\alpha_{1} i \partial_{1}+\alpha_{2} i \partial_{2}+\alpha_{3} i \partial_{3}=\sum_{i=1}^{3} \alpha_{i} i \partial_{i}=\alpha_{i} i \partial_{i}
$$

e chegamos à forma compacta da equação:

$$
i \partial_{0} \psi=\left(-\alpha_{i} i \partial_{i}+\beta m\right) \psi
$$

O lado direito da Eq. (16), bem como o da Eq. (11), representa o hamiltoniano de Dirac para a partícula livre:

$$
\hat{H}=\left(-\alpha_{i} i \partial_{i}+\beta m\right) .
$$

Sendo o hamiltoniano um operador hermitiano, as matrizes $\beta, \alpha_{i}$ também são hermitianas

$$
\beta^{\dagger}=\beta, \alpha_{i}^{\dagger}=\alpha_{i} .
$$


Multiplicando os termos da Eq. (16) por $\beta$ a esquerda, temos:

$$
\beta i \partial_{0} \psi=\left(-\beta \alpha_{i} i \partial_{i}+\beta^{2} m\right) \psi .
$$

Na Eq. (19) introduzimos para a matriz $\beta$ e para o produto $\beta \alpha_{i}$ a notação

$$
\beta=\gamma^{0}, \beta \alpha_{i}=\gamma^{i}
$$

e escrevemos todos os termos no lado esquerdo aplicando a condição $\beta^{2}=1$ da Eq. (10):

$$
\gamma^{0} i \partial_{0} \psi+\gamma^{i} i \partial_{i} \psi-m \psi=0 .
$$

Usando a convenção de somatório por índices repetidos, podemos reunir os dois primeiros termos na
Eq. (21) como

$$
\gamma^{0} i \partial_{0}+\gamma^{i} i \partial_{i}=\gamma^{\mu} i \partial_{\mu}
$$

Assim, chegamos ao aspecto usual da equação de Dirac que é usado comumente na teoria relativística:

$$
\left(\gamma^{\mu} i \partial_{\mu}-m\right) \psi=0
$$

As matrizes $\gamma^{\mu}$ também são chamadas de matrizes de Dirac.

Utilizando a Eq. 20 como definições das matrizes $\gamma^{\mu}$ e as condições para as matrizes $\beta, \alpha_{i}$ da Eq. (10), podemos obter as condições obedecidas pelas matrizes $\gamma^{\mu}$ :

$$
\begin{gathered}
\left(\gamma^{0}\right)^{2}=\beta^{2}=1, \\
\left(\gamma^{i}\right)^{2}=\beta \alpha_{i} \beta \alpha_{i}=-\beta \beta \alpha_{i} \alpha_{i}=-\beta^{2} \alpha_{i}^{2}=-1, \\
\gamma^{0} \gamma^{i}+\gamma^{i} \gamma^{0}=\beta \beta \alpha_{i}+\beta \alpha_{i} \beta=\beta^{2} \alpha_{i}-\beta \beta \alpha_{i}=\beta^{2} \alpha_{i}-\beta^{2} \alpha_{i}=0, \\
i \neq j, \gamma^{i} \gamma^{j}+\gamma^{j} \gamma^{i}=\beta \alpha_{i} \beta \alpha_{j}+\beta \alpha_{j} \beta \alpha_{i}=-\beta \beta \alpha_{i} \alpha_{j}-\beta \beta \alpha_{j} \alpha_{i}=-\beta^{2}\left(\alpha_{i} \alpha_{j}+\alpha_{j} \alpha_{i}\right)=0 .
\end{gathered}
$$

De forma compacta podemos escrever as expressões acima, como:

$$
\begin{aligned}
& \left(\gamma^{0}\right)^{2}=1,\left(\gamma^{i}\right)^{2}=-1, \gamma^{\mu} \gamma^{\nu}+\gamma^{\nu} \gamma^{\mu} \\
& =\left\{\gamma^{\mu}, \gamma^{\nu}\right\}=0, \mu \neq \nu,
\end{aligned}
$$

onde, as chaves $\{$,$\} designam o anticomutador.$

$\mathrm{Na}$ Eq. (14) para as coordenadas $x^{\mu}$ é usada uma notação de 4 -vetores em que a posição do índice é significativa: um 4-vetor com o índice em cima $x^{\mu}$ é chamado contravariante e um 4 -vetor com o índice em baixo $x_{\mu}$ é chamado covariante. A relação entre as componentes do 4-vetor contravariante e covariante é a seguinte:

$$
\left(x_{0}, x_{i}\right)=\left(x^{0},-x^{i}\right) .
$$

Escrevendo o somatório $x_{\mu} x^{\mu}$, explicitamente, temos:

$$
\begin{aligned}
& x_{\mu} x^{\mu}=\left(x^{0}\right)^{2}-\left(x^{1}\right)^{2}-\left(x^{2}\right)^{2}-\left(x^{3}\right)^{2} \\
& =\left(x^{0}\right)^{2}-\vec{x}^{2}
\end{aligned}
$$

vemos que ele representa um intervalo entre dois pontos do espaço-tempo. A energia e o momento de uma partícula também podem ser escritos na forma de um 4-vetor de energia-momento:

$$
\begin{aligned}
& p^{\mu}=\left(p^{0}, p^{1}, p^{2}, p^{3}\right)=\left(p^{0}, p^{i}\right) \\
& =\left(p^{0}, \vec{p}\right), i=1,2,3,
\end{aligned}
$$

onde $p^{0}$ denota energia relativística, $p^{0}=p_{0}=$ $\left(\vec{p}^{2}+m^{2}\right)^{1 / 2}$, e $\vec{p}$ é o momento relativístico. Para o somatório $p_{\mu} p^{\mu}$ temos

$$
\begin{aligned}
& p_{\mu} p^{\mu}=\left(p^{0}\right)^{2}-\left(p^{1}\right)^{2}-\left(p^{2}\right)^{2}-\left(p^{3}\right)^{2} \\
& =\left(p^{0}\right)^{2}-\vec{p}^{2}=m^{2}
\end{aligned}
$$

que representa uma expressão da energia de uma partícula relativística, a Eq. (5). Escrevemos na forma explícita mais um somatório que será usado no artigo

$$
p_{\mu} x^{\mu}=p_{0} x^{0}+p_{i} x^{i}=p_{0} x^{0}-\vec{p} \vec{x} .
$$

Com o uso da função de onda $\psi$ e das matrizes de Dirac $\gamma^{\mu}$, constrói-se uma quantidade $j^{\mu}$ chamada 
de 4-vetor da corrente de probabilidade:

$$
\begin{aligned}
& j^{\mu}=\bar{\psi} \gamma^{\mu} \psi=\left(j^{0}, j^{1}, j^{2}, j^{3}\right)=\left(j^{0}, j^{i}\right) \\
& =\left(j^{0}, \vec{j}\right), i=1,2,3,
\end{aligned}
$$

onde $\vec{j}$ representa o 3 -vetor da corrente de probabilidade e $j^{0}$ é a densidade de probabilidade. Na Eq. (30) o símbolo $\bar{\psi}$ denota a conjugação de Dirac de $\psi$ definida como:

$$
\bar{\psi}=\psi^{\dagger} \gamma^{0}
$$

onde o símbolo ${ }^{\dagger}$ é a conjugação hermitiana, que implica conjugação complexa * das componentes de $\psi$ e a sua transposição ${ }^{\top}$ :

$$
\psi^{\dagger}=\left(\psi^{*}\right)^{\top}=\left(\psi_{1}^{*}, \psi_{2}^{*}, \psi_{3}^{*}, \psi_{4}^{*}\right) .
$$

Particularmente, para a densidade de probabilidade $j^{0}$ temos:

$$
\begin{aligned}
& j^{0}=\bar{\psi} \gamma^{0} \psi=\psi^{\dagger} \gamma^{0} \gamma^{0} \psi=\psi^{\dagger} \psi \\
& =\left|\psi_{1}\right|^{2}+\left|\psi_{2}\right|^{2}+\left|\psi_{3}\right|^{2}+\left|\psi_{4}\right|^{2} .
\end{aligned}
$$

As matrizes de Dirac não são definidas de forma única, são utilizadas várias representações. As representações mais usadas são: a representação de Dirac

$$
\gamma^{0}=\left(\begin{array}{cc}
1_{2} & 0_{2} \\
0_{2} & -1_{2}
\end{array}\right), \gamma^{i}=\left(\begin{array}{cc}
0_{2} & \sigma^{i} \\
-\sigma^{i} & 0_{2}
\end{array}\right),
$$

a representação de Weyl ou representação quiral

$$
\gamma^{0}=\left(\begin{array}{ll}
0_{2} & 1_{2} \\
1_{2} & 0_{2}
\end{array}\right), \gamma^{i}=\left(\begin{array}{cc}
0_{2} & \sigma^{i} \\
-\sigma^{i} & 0_{2}
\end{array}\right)
$$

e a representação de Majorana

$$
\begin{aligned}
& \gamma^{0}=\left(\begin{array}{ll}
0_{2} & \sigma^{2} \\
\sigma^{2} & 0_{2}
\end{array}\right), \gamma^{1}=\left(\begin{array}{cc}
i \sigma^{3} & 0_{2} \\
0_{2} & i \sigma^{3}
\end{array}\right) \\
& \gamma^{2}=\left(\begin{array}{cc}
0_{2} & -\sigma^{2} \\
\sigma^{2} & 0_{2}
\end{array}\right), \gamma^{3}=\left(\begin{array}{cc}
-i \sigma^{1} & 0_{2} \\
0_{2} & -i \sigma^{1}
\end{array}\right)
\end{aligned}
$$

Nas Eqs. (34), (35) e (36) são usadas as notações: $1_{2}$ é a matriz unidade $2 \times 2,0_{2}$ é uma matriz $2 \times 2 \mathrm{com}$ todas as componentes nulas e $\sigma^{i}$ são as matrizes de Pauli:

$$
\begin{aligned}
\sigma^{1} & =\left(\begin{array}{cc}
0 & 1 \\
1 & 0
\end{array}\right), \sigma^{2}=\left(\begin{array}{cc}
0 & -i \\
i & 0
\end{array}\right), \\
\sigma^{3} & =\left(\begin{array}{cc}
1 & 0 \\
0 & -1
\end{array}\right) .
\end{aligned}
$$

Determinadas representações são úteis para tipos diferentes de estudos. Por exemplo, a representação de Dirac é mais conveniente para a discussão do limite não relativístico da equação de Dirac. O uso da representação de Weyl facilita o estudo de fenômenos de quiralidade de férmions. Na representação de Majorana todas as componentes das matrizes de Dirac $\gamma^{\mu}$ são imaginárias. Uma vantagem desta representação é que os spinores na equação são reais.

Além disso, as matrizes de Dirac e spinores nas representações diferentes são relacionados por uma transformação unitária, isso pode ser demonstrado da seguinte maneira: supomos que o spinor em uma representação $\psi$ e o spinor em outra representação $\widetilde{\psi}$, são relacionados por uma transformação não singular (inversível) $U$ :

$$
\widetilde{\psi}=U \psi
$$

A transformação $U$ é considerada como uma matriz complexa $4 \times 4$. As propriedades físicas da partícula não devem depender da representação usada. Particularmente a densidade de probabilidade, que é representada pela componente zero do 4 -vetor da corrente de probabilidade, deve satisfazer: $\widetilde{j}^{0}=j^{0}$. Das Eqs. (33) e (38), temos:

$$
\begin{aligned}
& j^{0}=\psi^{\dagger} \psi, \widetilde{j}^{0}=\widetilde{\psi}^{\dagger} \widetilde{\psi}=\psi^{\dagger} U^{\dagger} U \psi \\
& \Rightarrow \psi^{\dagger} \psi=\psi^{\dagger} U^{\dagger} U \psi,
\end{aligned}
$$

portanto,

$$
U^{\dagger} U=1,
$$

isto é, a matriz $U$ é unitária. A equação de Dirac na representação til tem a forma

$$
\left(\widetilde{\gamma}^{\mu} i \partial_{\mu}-m\right) \widetilde{\psi}=0
$$

onde $\widetilde{\gamma}^{\mu}$ são as matrizes de Dirac nesta representação. Demostraremos qual é a relação entre as matrizes de Dirac nas representações diferentes. Com o uso das Eqs. 38 , 40 escrevemos $\psi$ como $\psi=U^{\dagger} \widetilde{\psi}$ e substituimos na Eq. (23):

$$
\gamma^{\mu} U^{\dagger} i \partial_{\mu} \widetilde{\psi}-m U^{\dagger} \widetilde{\psi}=0 .
$$

Muiltiplicamos os termos da Eq. 42 por $U$ a esquerda. Chegamos ao resultado:

$$
U \gamma^{\mu} U^{\dagger} i \partial_{\mu} \widetilde{\psi}-m U U^{\dagger} \widetilde{\psi}=0
$$

Sendo $U$ unitária, isto é, $U^{\dagger} U=U U^{\dagger}=1$, temos

$$
\left(U \gamma^{\mu} U^{\dagger} i \partial_{\mu}-m\right) \widetilde{\psi}=0 .
$$

Comparando a Eq. 43 com a Eq. 41] vemos que

$$
\widetilde{\gamma}^{\mu}=U \gamma^{\mu} U^{\dagger}
$$

As matrizes de Dirac $\widetilde{\gamma}^{\mu}$ possuem as mesmas propriedades das matrizes $\gamma^{\mu}$ da Eq. (24). Os quadrados das matrizes $\widetilde{\gamma}^{\mu}$ são 


$$
\begin{aligned}
& \left(\widetilde{\gamma}^{0}\right)^{2}=U \gamma^{0} U^{\dagger} U \gamma^{0} U^{\dagger}=U \gamma^{0} \gamma^{0} U^{\dagger}=U\left(\gamma^{0}\right)^{2} U^{\dagger}=U U^{\dagger}=1 \\
& \left(\widetilde{\gamma}^{i}\right)^{2}=U \gamma^{i} U^{\dagger} U \gamma^{i} U^{\dagger}=U \gamma^{i} \gamma^{i} U^{\dagger}=U\left(\gamma^{i}\right)^{2} U^{\dagger}=-U U^{\dagger}=-1
\end{aligned}
$$

e elas anticomutam, se $\mu \neq \nu$,

$$
\begin{aligned}
\left\{\widetilde{\gamma}^{\mu}, \widetilde{\gamma}^{\nu}\right\} & =\widetilde{\gamma}^{\mu} \widetilde{\gamma}^{\nu}+\widetilde{\gamma}^{\nu} \widetilde{\gamma}^{\mu}=U \gamma^{\mu} U^{\dagger} U \gamma^{\nu} U^{\dagger}+U \gamma^{\mu} U^{\dagger} U \gamma^{\nu} U^{\dagger} \\
& =U \gamma^{\mu} \gamma^{\nu} U^{\dagger}+U \gamma^{\mu} \gamma^{\nu} U^{\dagger}=U\left(\gamma^{\mu} \gamma^{\nu}+\gamma^{\mu} \gamma^{\nu}\right) U^{\dagger}=U\left\{\gamma^{\mu}, \gamma^{\nu}\right\} U^{\dagger}=0
\end{aligned}
$$

Para demonstrar como as matrizes $\beta, \alpha_{i}$ são relacionadas em representações diferentes, usamos a Eq. (44) para as matrizes $\gamma^{\mu}$ :

$$
\begin{gathered}
\widetilde{\beta}=\widetilde{\gamma}^{0}=U \gamma^{0} U^{\dagger}=U \beta U^{\dagger} \\
\widetilde{\alpha}_{i}=\widetilde{\gamma}^{0} \widetilde{\gamma}^{i}=U \gamma^{0} U^{\dagger} U \gamma^{i} U^{\dagger}=U \gamma^{0} \gamma^{i} U^{\dagger}=U \alpha_{i} U^{\dagger} .
\end{gathered}
$$

Escrevemos ainda a equação de Dirac na forma hamiltoniana, para uma partícula livre, usando o hamiltoniano da Eq. (17):

$$
\hat{H} \psi=\left(-\alpha_{i} i \partial_{i}+\beta m\right) \psi=\varepsilon \psi, \varepsilon=\text { const }
$$

onde $\varepsilon$ denota a energia que é positiva no caso de partícula $\varepsilon=\varepsilon_{+}=p_{0}>0$ e negativa no caso de antipartícula $\varepsilon=\varepsilon_{-}=-p_{0}<0$, onde $p_{0}=\left(p_{i}^{2}+m^{2}\right)^{1 / 2}$. Para a função de onda da partícula livre da Eq. (16) temos:

$$
i \partial_{0} \psi=\varepsilon \psi \Rightarrow \psi\left(x^{\mu}\right)=e^{-i \varepsilon x^{0}} \psi\left(x^{i}\right) .
$$

Escrevemos separadamente a função de onda para partículas

$$
\psi_{+}\left(x^{\mu}\right)=e^{-i p_{0} x^{0}} \psi_{+}\left(x^{i}\right)
$$

e para antipartículas

$$
\psi_{-}\left(x^{\mu}\right)=e^{i p_{0} x^{0}} \psi_{-}\left(x^{i}\right) .
$$

\section{A equação de Dirac em Baixas Dimensões}

Em livros e artigos são indicadas várias motivações para os estudos da teoria quântica relativística em baixas dimensões. Na nossa opinião, a motivação mais condensada e elegante é formulada na Ref. [10]. Citamos diretamente um parágrafo da Ref. 10. "Em baixas dimensões de espaço-tempo, $1+1$ e $2+1$, a teoria quântica relativística exibe especificidades estruturais que são ausentes em dimensões $3+1$ de espaço-tempo. Acontece que estas especificidades não são somente curiosidades não físicas de "toy models", mas são de grande interesse em aplicações na área de física da matéria condensada e podem ter um impacto na área de física de partículas, fornecendo idéias novas para a construção de modelos."

Nas baixas dimensões, com uma dimensão espacial $(1+1)$ ou com duas dimensões espaciais $(2+1)$, as matrizes de Dirac são representadas por matrizes complexas $2 \times 2$. Particularmente, as matrizes $\beta$, $\alpha_{i}$ podem ser definidas por matrizes de Pauli da Eq. (37), porque as matrizes de Pauli obedecem às condições necessárias para matrizes de Dirac da Eq. (10). Uma certa escolha das matrizes de Pauli determina uma representação das matrizes de Dirac e a correspondente representação do spinor. Nas baixas dimensões é fácil estimar o número total de representações distintas das matrizes de Dirac. No caso de $1+1$ dimensões a equação de Dirac possui somente duas matrizes: $\beta$ e $\alpha$. O número de opções da escolha de dois elementos do conjunto de três elementos é 3. Se a ordem dos dois elementos determina opções diferentes, o número das opções é dobrado: $2 \cdot 3=6$. Além disso, uma matriz de Pauli associada à matriz de Dirac pode ter sinal positivo ou negativo, o que multiplica o número das opções por quatro. Então, o número total das representações em $1+1$ dimensões é determinado como $6 \cdot 4=24$. No caso de $2+1$ dimensões a equação de Dirac possui três matrizes: $\beta, \alpha_{1}$ e $\alpha_{2}$. O número de opções de escolha de três elementos do conjunto de três elementos, levando em conta a ordem dos elementos, é 6. Além disso, uma matriz de Pauli associada à matriz de Dirac pode ter sinal positivo ou negativo, multiplicando o número das opções por 8. Então, o número total das representações em $2+1$ dimensões é determinado 
como $6 \cdot 8=48$. Como vemos, o número de representações distintas da equação de Dirac nas baixas dimensões não é grande, permitindo fazer uma descrição completa das representações com o uso de ferramentas matemáticas bastante simples. Achamos que este tipo de investigação é compreensível a alunos de graduação que possuem conhecimento acerca de conceitos básicos de relatividade especial e da mecânica quântica e com intenção de especialização na área de física teórica.

Neste trabalho consideraremos a equação de Dirac em 1+1 dimensões. Propomos uma classificação unificada das representações e determinaremos todas as transformações unitárias entre elas na forma explícita. A estrutura algébrica do conjunto das transformações será estudada. Apresentaremos a forma mais geral das matrizes de Dirac em 1+1 dimensões e discutiremos isomorfismos destas matrizes. Apresentaremos também algumas aplicações dos resultados obtidos neste trabalho.

Foi observado que, em trabalhos envolvendo a equação de Dirac em 1+1 dimensões, são usadas várias representações e em alguns casos surge uma necessidade de transição entre representações. Vemos que uma descrição completa das representações pode ser usada como um ponto de referência para estudos nesse tema. Mencionamos alguns trabalhos onde são usadas diferentes representações. Por exemplo, no trabalho [11] é discutida a equação de Dirac em $1+1$ dimensões com potencial elétrico $V_{0}=-q|x|$. Para a busca de soluções da equação, é usada a representação das matrizes de Dirac $\beta=\sigma^{1}, \alpha=\sigma^{3}$, mas para a discussão do limite não relativístico é usada a representação $\beta=\sigma^{3}, \alpha=\sigma^{1}$, chamada de representação "padrão"porque $\beta$ tem forma diagonal nesta representação. Uma transformação entre as duas representações é indicada de forma implícita no trabalho [11]. No trabalho 12 é considerada a equação de Dirac em $1+1$ dimensões com potencial escalar $V_{s}=g|x|$. Para a busca de soluções da equação, é usada a representação das matrizes de Dirac $\beta=\sigma^{1}, \alpha=\sigma^{2}$, chamada por alguns autores, como o da Ref. [13], por exemplo, de representação de Jackiw-Rebbi. Para a discussão do limite não relativístico é usada a representação $\beta=\sigma^{3}, \alpha=\sigma^{2}$. No trabalho 12 é apresentada também uma transformação unitária entre as duas representações na forma explícita $U=\frac{i}{\sqrt{2}}\left(\begin{array}{cc}1 & 1 \\ -1 & 1\end{array}\right)$. No artigo 14 é discutida a equação de Dirac com potencial confinante e indicado que a representação $\beta=\sigma^{1}, \alpha=\sigma^{2}$ é mais conveniente para discussão de aspectos supersimétricos, mas a representação $\beta=\sigma^{3}, \alpha=\sigma^{2}$ é mais útil para observação de relações entre os casos de $1+1$, $2+1$ e $3+1$ dimensões. No artigo [15] são discutidas as condições de contorno para uma partícula de Dirac em uma caixa unidimensional. No trabalho são usadas a representação $\beta=\sigma^{3}, \alpha=\sigma^{1}$, chamada de representação de Dirac e a representação $\beta=\sigma^{1}, \alpha=\sigma^{3}$ chamada de representação de Weyl. No trabalho é indicado também que para discussão do limite não relativístico é mais conveniente a utilização da representação de Dirac.

Outra motivação do nosso trabalho é o interesse atual no tema da equação de Dirac em $1+1$ dimensões. Recentemente a equação de Dirac, ou a equação do tipo Dirac em $1+1$ dimensões, são ativamente utilizadas em estudos nas áreas de matéria condensada e física atômica e molecular, para a descrição de sistemas em baixas dimensões que demonstram comportamento relativístico de partículas ou quasi-partículas. Mencionamos alguns trabalhos recentes desta linha de pesquisa. No trabalho 16 são consideradas as partículas de Dirac em uma dimensão com massa e velocidade dependentes da posição. É demonstrado que as soluções do tipo estados ligados no contínuo são possíveis apenas quando uma relação especial entre massa e velocidade das partículas é satisfeita. No trabalho [17], é estudado um hamiltoniano unidimensional do tipo Dirac, com massa e velocidade na forma de degrau. São determinadas as extensões auto-adjuntas físicas desse hamiltoniano e são apresentados os seus espectros. É discutida uma transição através de heteroestruturas unidimensionais descritas pela equação de Dirac. No artigo [18, é demonstrado que o limite de confinamento da partícula de Dirac de uma dimensão espacial sujeita ao potencial vetorial e escalar é $\lambda_{C} / \sqrt{2}$, onde $\lambda_{C}$ é comprimento de onda Compton da partícula. A validade do limite de confinamento é estendida para os potenciais não simétricos. No artigo [19, é construído o operador de "time of arrival" conjugado ao hamiltoniano que descreve a partícula relativística de spin $1 / 2$ de uma dimensão espacial. No artigo é demostrado que o operador construído consiste de um termo $\widehat{T}$ cuja ação é a mesma do caso de spin-0 e outro termo $\widehat{T}_{0}$ que comuta com o hamiltoniano. Impondo a condição de invariância relativa a inversão da paridade no 
operador de "time of arrival", o operador contém somente o termo $\widehat{T}$. No trabalho [20], é considerada a equação de Dirac em $1+1$ dimensões com potenciais mistos vetorial-escalar-pseudoescalar da forma quadrada, que representam a mais geral estrutura de Lorentz do potencial matricial. É demonstrado que a existência de estados ligados é condicionada pela magnitude do potencial pseudoescalar que possui um valor crítico.

\section{A Equação de Dirac em 1+1 Dimensões}

Apresentamos a equação de Dirac em $1+1$ dimensões na forma hamiltoniana

$$
\hat{H} \psi=\left[-i \alpha \partial_{1}+\beta m\right] \psi=\varepsilon \psi,
$$

onde são usadas as unidades naturais, $\alpha, \beta$ são as matrizes de Dirac e $\partial_{1}=\partial / \partial x^{1}$. A Eq. (53) descreve tanto partículas quanto antipartículas. A solução da Eq. (53) para partículas pode ser escrita como:

$$
\psi_{+}=e^{-i p x} u_{p}, \varepsilon_{+}=p_{0}=\sqrt{m^{2}+p_{1}^{2}}
$$

e para antipartículas como:

$$
\psi_{-}=e^{i p x} v_{p}, \varepsilon_{-}=-p_{0}=-\sqrt{m^{2}+p_{1}^{2}},
$$

onde $p x=p_{\mu} x^{\mu}=p_{0} x^{0}+p_{1} x^{1}$ e $u_{p}, v_{p}$ são spinores constantes que obedecem às equações:

$$
\left[-\alpha p_{1}+\beta m\right] u_{p}=\varepsilon_{+} u_{p},\left[\alpha p_{1}+\beta m\right] v_{p}=\varepsilon_{-} v_{p}
$$

As matrizes de Dirac $\beta, \alpha$ não são definidas de forma única. Uma das escolhas das matrizes de Dirac determina uma certa representação. Os spinores de Dirac nas representações distintas são relacionados por uma transformação unitária $U$ :

$$
\widetilde{\psi}=U \psi, U^{\dagger} U=1
$$

Se $\psi$ obedece a equação de Dirac $(53)$, então $\widetilde{\psi}$ obedece a equação

$$
\left[-i \widetilde{\alpha} \partial_{1}+\widetilde{\beta} m\right] \widetilde{\psi}=\varepsilon \widetilde{\psi}
$$

onde

$$
\widetilde{\beta}=U \beta U^{\dagger}, \widetilde{\alpha}=U \alpha U^{\dagger} .
$$

Para partículas e antipartículas temos as relações entre as representações distintas:

$$
\begin{aligned}
& \widetilde{\psi}_{+}=U \psi_{+}=e^{-i p x} U u_{p}=e^{-i p x} \widetilde{u}_{p}, \widetilde{u}_{p}=U u_{p}, \\
& \widetilde{\psi}_{-}=U \psi_{-}=e^{i p x} U v_{p}=e^{i p x} \widetilde{v}_{p}, \widetilde{v}_{p}=U v_{p} .
\end{aligned}
$$

As matrizes de Dirac $\beta$, $\alpha$ são hermitianas

$$
\beta^{\dagger}=\beta, \alpha^{\dagger}=\alpha
$$

e obedecem as relações

$$
\beta^{2}=\alpha^{2}=1,\{\alpha, \beta\}=\alpha \beta+\beta \alpha=0 .
$$

As condições 60, (61) são obedecidas particularmente pelas matrizes de Pauli 1 da Eq. (37). As duas matrizes de Dirac $\beta, \alpha$ podem ser escolhidas arbitrariamente entre as três matrizes de Pauli. Uma certa escolha das matrizes determina uma representação de matrizes de Dirac e a correspondente representação do spinor.

\section{Notação e Classificação de Representações}

Apresentamos todas as representações das matrizes de Dirac na forma explícita como:

$$
\begin{aligned}
& \beta=s \sigma^{i}, \alpha=t \sigma^{j} ; s= \pm 1, t= \pm 1 \\
& i, j=1,2,3 ; i \neq j
\end{aligned}
$$

As representações definidas por $\beta=\sigma^{i}$ e $\alpha=\sigma^{j}$ $\operatorname{com} \varepsilon_{i j k}=+1$, denominaremos de representações cíclicas, e com $\varepsilon_{i j k}=-1$, denominaremos de representações anticíclicas, sendo $\varepsilon_{i j k}$ o símbolo de Levi-Civita, $\varepsilon_{123}=+1$. As representações com valores $s=+1$ e $t=+1$ da Eq. 62 , denominaremos de representações principais. As representações com $s=-1 \mathrm{e} /$ ou $t=-1$ chamaremos de representações não principais. Para as matrizes de Dirac $\beta, \alpha$ e spinores $\psi$ em uma certa representação usaremos a notação:

$$
\beta^{\left[i_{q}\right]}, \alpha^{\left[i_{q}\right]} ; u_{p}^{\left[i_{q}\right]}, v_{p}^{\left[i_{q}\right]},
$$

onde o índice $i$ representa um quarteto de representações em que $\beta, \alpha$ são associadas às mesmas matrizes de Pauli. Para as representações cíclicas utilizaremos $i=1,2,3$ e para as representações anticíclicas utilizaremos $i=4,5,6$, conforme a Tabela (1). O subíndice $q$ indica as representações

\footnotetext{
${ }^{1} \mathrm{O}$ caso mais geral da forma das matrizes de Dirac é discutida separadamente no Apêndice.
} 
Tabela 1: Representações principais: cíclicas e anticíclicas.

\begin{tabular}{ccccc}
\hline $\begin{array}{c}\text { Representações } \\
\text { cíclicas }\end{array}$ & $\beta$ & $\alpha$ & $\gamma^{0}=\beta$ & $\gamma^{1}=\beta \alpha$ \\
\hline 1 & $\sigma^{1}$ & $\sigma^{2}$ & $\sigma^{1}$ & $i \sigma^{3}$ \\
2 & $\sigma^{2}$ & $\sigma^{3}$ & $\sigma^{2}$ & $i \sigma^{1}$ \\
3 & $\sigma^{3}$ & $\sigma^{1}$ & $\sigma^{3}$ & $i \sigma^{2}$ \\
\hline
\end{tabular}

\begin{tabular}{ccccc}
\hline $\begin{array}{c}\text { Representações } \\
\text { anticíclicas }\end{array}$ & $\beta$ & $\alpha$ & $\gamma^{0}=\beta$ & $\gamma^{1}=\beta \alpha$ \\
\hline 4 & $\sigma^{1}$ & $\sigma^{3}$ & $\sigma^{1}$ & $-i \sigma^{2}$ \\
5 & $\sigma^{2}$ & $\sigma^{1}$ & $\sigma^{2}$ & $-i \sigma^{3}$ \\
6 & $\sigma^{3}$ & $\sigma^{2}$ & $\sigma^{3}$ & $-i \sigma^{1}$ \\
\hline
\end{tabular}

não principais, $\operatorname{com} q=1,2,3$, o valor $q=0$ é reservado para representações principais, conforme a Tabela (2). Para as manipulações com objetos das representações principais utilizaremos uma notação simplificada sem o subíndice "0": $\beta^{[i]}, \alpha^{[i]} ; u_{p}^{[i]}, v_{p}^{[i]}$.

Tabela 2: Representações não principais.

\begin{tabular}{ccc}
\hline$q$ & $s$ & $t$ \\
\hline 0 & +1 & +1 \\
1 & +1 & -1 \\
2 & -1 & +1 \\
3 & -1 & -1 \\
\hline
\end{tabular}

\section{Transformações Unitárias entre Representações}

Nesta seção determinaremos as transformações unitárias entre as várias representações da forma 62. Para uma transformação de $u_{p}^{\left[i_{q}\right]}$ para $u_{p}^{\left[j_{r}\right]}$ usaremos a notação $U_{j_{r}, i_{q}}: u_{p}^{\left[i_{q}\right]} \rightarrow u_{p}^{\left[j_{r}\right]}$, tal que:

$$
u_{p}^{\left[j_{r}\right]}=U_{j_{r}, i_{q}} u_{p}^{\left[i_{q}\right]} .
$$

Indicaremos algumas propriedades das transformações. Para uma escrita compacta das fórmulas, utilizaremos uma notação condensada do símbolo das representações: $\left[i_{q}\right]=[a],\left[j_{r}\right]=[b], \ldots$. Escrevemos uma regra de composição de duas transformações. Sendo

$$
u_{p}^{[c]}=U_{c b} u_{p}^{[b]}, u_{p}^{[b]}=U_{b a} u_{p}^{[a]},
$$

obtem-se

$$
u_{p}^{[c]}=U_{c b} U_{b a} u_{p}^{[a]} .
$$

Por outro lado, ao utilizarmos a expressão

$$
u_{p}^{[c]}=U_{c a} u_{p}^{[a]},
$$

chegamos a

$$
U_{c a}=U_{c b} U_{b a} .
$$

Indicamos que a transformação do tipo $U_{a a}$ representa uma transformação identidade que é representada por uma matriz unidade.
Para a conjugação hermitiana das matrizes das transformações temos:

$$
u_{p}^{[b]}=U_{b a} u_{p}^{[a]},
$$

portanto

$$
U_{b a}^{\dagger} u_{p}^{[b]}=u_{p}^{[a]} .
$$

De outro modo

$$
u_{p}^{[a]}=U_{a b} u_{p}^{[b]}
$$

portanto

$$
U_{a b}=U_{b a}^{\dagger} .
$$

Com o uso da regra de composição das transformações (63) podemos escrever no caso geral:

$$
U_{j_{r} i_{q}}=U_{j_{r} j_{0}} U_{j_{0} i_{0}} U_{i_{0} i_{q}} .
$$

Então, para determinar as transformações entre duas representações no caso geral, é suficiente determinar separadamente as transformações entre todas as representações principais, as transformações do tipo $U_{j_{0} i_{0}}$, e as transformações entre uma representação principal e as representações não principais dentro de um quarteto, isto é, as transformações do tipo $U_{i_{0} i_{q}}$ e $U_{j_{r} j_{0}}$.

Para encontrar as transformações na forma explícita, decompomos $U$ na base $\left(\sigma^{0}, \sigma^{i}\right)$, onde $\sigma^{i}$ são as matrizes de Pauli e $\sigma^{0}$ é a matriz unidade $2 \times 2$ :

$$
\begin{aligned}
U & =a_{\mu} \sigma^{\mu}=a_{0} \sigma^{0}+a_{i} \sigma^{i} \\
& =\left(\begin{array}{cc}
a_{0}+a_{3} & a_{1}-i a_{2} \\
a_{1}+i a_{2} & a_{0}-a_{3}
\end{array}\right), a_{\mu} \in \mathbb{C} .
\end{aligned}
$$

Nesta decomposição a matriz $U$ é definida por 4 coeficientes $a_{\mu}$. Juntando os coeficientes $a_{\mu}$ em um objeto de 4 componentes, escreveremos também $U$ na forma:

$$
U=\left(a_{\mu}\right)=\left(a_{0}, a_{1}, a_{2}, a_{3}\right)=\left(a_{0}, a_{i}\right)=\left(a_{0}, \vec{a}\right) .
$$

Sendo $U$ unitária, $U^{\dagger} U=1$, os coeficientes $a_{\mu}$ devem satisfazer as condições:

$$
\begin{aligned}
& \left|a_{0}\right|^{2}+\vec{a}^{*} \cdot \vec{a}=\left|a_{0}\right|^{2}+\left|a_{1}\right|^{2} \\
& +\left|a_{2}\right|^{2}+\left|a_{3}\right|^{2}=1,
\end{aligned}
$$




$$
\begin{aligned}
& a_{0}^{*} a_{j}+a_{j}^{*} a_{0}+a_{n}^{*} a_{k} i \varepsilon_{n k j} \\
& =a_{0}^{*} \vec{a}+\vec{a}^{*} a_{0}+i \vec{a}^{*} \times \vec{a}=0 .
\end{aligned}
$$

Apresentaremos também as seguintes relações úteis para cálculos:

$$
\begin{gathered}
U \sigma^{1}=a_{0} \sigma^{1}+a_{1}-i a_{2} \sigma^{3}+i a_{3} \sigma^{2}=\left(a_{1}, a_{0}, i a_{3},-i a_{2}\right), \\
U \sigma^{2}=a_{0} \sigma^{2}+i a_{1} \sigma^{3}+a_{2}-i a_{3} \sigma^{1}=\left(a_{2},-i a_{3}, a_{0}, i a_{1}\right), \\
U \sigma^{3}=a_{0} \sigma^{3}-i a_{1} \sigma^{2}+i a_{2} \sigma^{1}+a_{3}=\left(a_{3}, i a_{2},-i a_{1}, a_{0}\right), \\
\sigma^{1} U=a_{0} \sigma^{1}+a_{1}+i a_{2} \sigma^{3}-i a_{3} \sigma^{2}=\left(a_{1}, a_{0},-i a_{3}, i a_{2}\right), \\
\sigma^{2} U=a_{0} \sigma^{2}-i a_{1} \sigma^{3}+a_{2}+i a_{3} \sigma^{1}=\left(a_{2}, i a_{3}, a_{0},-i a_{1}\right), \\
\sigma^{3} U=a_{0} \sigma^{3}+i a_{1} \sigma^{2}-i a_{2} \sigma^{1}+a_{3}=\left(a_{3},-i a_{2}, i a_{1}, a_{0}\right), \\
{\left[\sigma^{1}, U\right]=2 i a_{2} \sigma^{3}-2 i a_{3} \sigma^{2},\left[\sigma^{2}, U\right]=-2 i a_{1} \sigma^{3}+2 i a_{3} \sigma^{1},\left[\sigma^{3}, U\right]=2 i a_{1} \sigma^{2}-2 i a_{2} \sigma^{1},} \\
\left\{\sigma^{1}, U\right\}=2 a_{0} \sigma^{1}+2 a_{1},\left\{\sigma^{2}, U\right\}=2 a_{0} \sigma^{2}+2 a_{2},\left\{\sigma^{3}, U\right\}=2 a_{0} \sigma^{3}+2 a_{3},
\end{gathered}
$$

onde, os colchetes [,] designam o comutador, definido por $[A, B]=A B+B A$.

\subsection{Transformações entre Representações Principais}

Primeiramente consideraremos as transformações entre as representações principais. Para a determinação da forma explícita das transformações, usaremos as Eqs. 57) escritas como:

$$
{ }^{\beta^{[j]}} U_{j i}=U_{j i} \beta^{[i]}, \alpha^{[j]} U_{j i}=U_{j i} \alpha^{[i]} .
$$

Dividimos este problema em duas etapas. Na primeira etapa determinaremos as transformações entre as representações dentro do setor cíclico e anticíclico, na segunda etapa determinaremos as transformações entre as representações cíclicas e anticíclicas.

\subsubsection{Transformações do Setor Cíclico (Anticíclico)}

Iniciamos com a determinação das transformações entre as representações do setor cíclico. As transformações entre as representações do setor anticíclico serão obtidas como consequência dos cálculos. Para a determinação da transformação $U_{21}: u_{p}^{[1]} \rightarrow u_{p}^{[2]}$ da Eq. (73) e da Tabela (1) temos:

$$
U_{21}: \sigma^{2} U=U \sigma^{1}, \sigma^{3} U=U \sigma^{2} \text {. }
$$

Com o uso das Eqs. 70 obtemos:

$$
\begin{aligned}
& \left(a_{2}, i a_{3}, a_{0},-i a_{1}\right)=\left(a_{1}, a_{0}, i a_{3},-i a_{2}\right), \\
& \left(a_{3},-i a_{2}, i a_{1}, a_{0}\right)=\left(a_{2},-i a_{3}, a_{0}, i a_{1}\right),
\end{aligned}
$$

que resulta em

$$
a_{0}=i a_{3}, a_{1}=a_{2} \text { e } a_{0}=i a_{1}, a_{3}=a_{2},
$$

portanto

$$
a_{1}=a_{2}=a_{3}=-i a_{0} .
$$

Para satisfazer a Eq. (68) temos:

$$
4\left|a_{0}\right|^{2}=1 \Rightarrow a_{0}=\frac{1}{2} e^{i \varphi}
$$

onde $e^{i \varphi}$ é um fator de fase arbitrário, portanto

$$
U_{21}=\frac{1}{2} e^{i \varphi}(1,-i,-i,-i)
$$

Substituindo os coeficientes das Eqs. (75), (76) na Eq. (66), obtemos:

$$
\begin{gathered}
U_{21}=\frac{1}{2} e^{i \varphi}\left(\begin{array}{cc}
1-i & -i-1 \\
-i+1 & 1+i
\end{array}\right)=\frac{1}{2} e^{i \varphi}\left(\begin{array}{cc}
\sqrt{2} e^{-i \pi / 4} & -\sqrt{2} e^{i \pi / 4} \\
\sqrt{2} e^{-i \pi / 4} & \sqrt{2} e^{i \pi / 4}
\end{array}\right) \\
=\frac{1}{2} e^{i \varphi} \sqrt{2} e^{-i \pi / 4}\left(\begin{array}{cc}
1 & -e^{i \pi / 2} \\
1 & e^{i \pi / 2}
\end{array}\right)=\frac{1}{\sqrt{2}} e^{i \varphi} e^{-i \pi / 4}\left(\begin{array}{cc}
1 & -i \\
1 & i
\end{array}\right) .
\end{gathered}
$$


Impondo $\varphi=\pi / 4$, temos

$U_{21}=\frac{1}{2} e^{i \pi / 4}(1,-i,-i,-i)=\frac{1}{\sqrt{2}}\left(\begin{array}{cc}1 & -i \\ 1 & i\end{array}\right)=U_{0}$.

Na Eq. (77) é introduzida também a notação $U_{0}$ para esta matriz, a qual será usada nos cálculos futuros.

A matriz inversa é determinada pela Eq. (64):

$$
\begin{aligned}
U_{12} & =U_{21}^{\dagger}=\frac{1}{\sqrt{2}}\left(\begin{array}{cc}
1 & 1 \\
i & -i
\end{array}\right) \\
& =\frac{1}{2} e^{-i \pi / 4}(1, i, i, i)=U_{0}^{\dagger} .
\end{aligned}
$$

Notamos que a Eq. (74) escrita na forma

$$
\sigma^{3} U=U \sigma^{2}, \sigma^{2} U=U \sigma^{1}
$$

pode ser interpretada como a transformação $U_{65}$ : $u_{p}^{[5]} \rightarrow u_{p}^{[6]}$ do setor anticíclico, portanto

$$
U_{65}=U_{21}=U_{0}, U_{56}=U_{65}^{\dagger}=U_{0}^{\dagger} .
$$

Para a determinação da transformação $U_{32}$ : $u_{p}^{[2]} \rightarrow u_{p}^{[3]}$ da Eq. 73 e da Tabela (1) temos:

$$
U_{32}: \sigma^{3} U=U \sigma^{2}, \sigma^{1} U=U \sigma^{3} .
$$

A Eq. (80) escrita na forma

$$
\sigma^{1} U=U \sigma^{3}, \sigma^{3} U=U \sigma^{2},
$$

pode ser interpretada também como a transformação $U_{46}: u_{p}^{[6]} \rightarrow u_{p}^{[4]}$ do setor anticíclico.

Para a determinação da transformação $U_{13}$ : $u_{p}^{[3]} \rightarrow u_{p}^{[1]}$ da Eq. 73 e da Tabela (1) temos:

$$
U_{13}: \sigma^{1} U=U \sigma^{3}, \sigma^{2} U=U \sigma^{1} .
$$

A Eq. (81) escrita na forma

$$
\sigma^{2} U=U \sigma^{1}, \sigma^{1} U=U \sigma^{3},
$$

pode ser interpretada como a transformação $U_{54}$ : $u_{p}^{[4]} \rightarrow u_{p}^{[5]}$ do setor anticíclico.

Realizando cálculos semelhantes aos anteriores, chegamos à mesma condição da Eq. 75 para os coeficientes $a_{\mu}$, portanto

$$
U_{32}=U_{13}=U_{0}, U_{23}=U_{31}=U_{0}^{\dagger} .
$$

e

$$
U_{46}=U_{54}=U_{0}, U_{64}=U_{45}=U_{0}^{\dagger} .
$$

Notamos que as transformações são determinadas a menos de um fator de fase arbitrário. Para refletir este fato, em vez do sinal de igualdade usaremos o símbolo $\simeq$ que significará igualdade a menos de um fator de fase arbitrário. Unimos os resultados dos cálculos das transformações do setor cíclico e anticíclico em expressões compactas:

$$
\begin{aligned}
& U_{21} \simeq U_{32} \simeq U_{13} \simeq U_{54} \simeq U_{65} \simeq U_{46} \simeq U_{0} \\
& =\frac{1}{\sqrt{2}}\left(\begin{array}{cc}
1 & -i \\
1 & i
\end{array}\right) \simeq \frac{1}{2}(1,-i,-i,-i), \\
& U_{12} \simeq U_{23} \simeq U_{31} \simeq U_{45} \simeq U_{56} \simeq U_{64} \simeq U_{0}^{\dagger} \\
& =\frac{1}{\sqrt{2}}\left(\begin{array}{cc}
1 & 1 \\
i & -i
\end{array}\right) \simeq \frac{1}{2}(1, i, i, i) .
\end{aligned}
$$

Notamos que, a partir da regra de composição da Eq. 63), temos

$$
U_{21} U_{13}=U_{23}, U_{12} U_{23}=U_{13},
$$

portanto, das Eqs. (84) e (85), obtemos

$$
U_{0}^{2} \simeq U_{0}^{\dagger}, U_{0}^{\dagger 2} \simeq U_{0} .
$$

\subsubsection{Transformações entre Representações Cíclicas e Anticíclicas}

Determinaremos as transformações entre as representações cíclicas e anticíclicas. Iniciamos com a transformação $U_{26}: u_{p}^{[6]} \rightarrow u_{p}^{[2]}$. Da Eq. 73 e da Tabela (1) temos:

$$
U_{26}: \sigma^{2} U=U \sigma^{3}, \sigma^{3} U=U \sigma^{2} .
$$

Usando as Eqs. (70), temos

$$
\begin{aligned}
& \left(a_{2}, i a_{3}, a_{0},-i a_{1}\right)=\left(a_{3}, i a_{2},-i a_{1}, a_{0}\right), \\
& \left(a_{3},-i a_{2}, i a_{1}, a_{0}\right)=\left(a_{2},-i a_{3}, a_{0}, i a_{1}\right),
\end{aligned}
$$

que resulta em

$$
a_{0}=-i a_{1}, a_{2}=a_{3} \text { e } a_{0}=i a_{1}, i a_{2}=i a_{3},
$$

portanto

$$
a_{0}=a_{1}=0, a_{2}=a_{3} .
$$

Para satisfazer a Eq. (68) temos

$$
2\left|a_{2}\right|^{2}=1 \Rightarrow a_{2}=a_{3}=\frac{e^{i \varphi}}{\sqrt{2}} .
$$

Impondo $\varphi=0$, obtemos

$$
U_{26}=\frac{1}{\sqrt{2}}(0,0,1,1)=\frac{1}{\sqrt{2}}\left(\begin{array}{cc}
1 & -i \\
i & -1
\end{array}\right) .
$$


Notamos que

$$
U_{26}=U_{26}^{\dagger}=U_{62}
$$

Para a determinação da transformação $U_{34}$ : $u_{p}^{[4]} \rightarrow u_{p}^{[3]}$ da Eq. 73 e da Tabela (1) temos:

$$
U_{34}: \sigma^{3} U=U \sigma^{1}, \sigma^{1} U=U \sigma^{3} .
$$

Com o uso das Eqs. (70), temos:

$$
\begin{aligned}
& \left(a_{3},-i a_{2}, i a_{1}, a_{0}\right)=\left(a_{1}, a_{0}, i a_{3},-i a_{2}\right), \\
& \left(a_{1}, a_{0},-i a_{3}, i a_{2}\right)=\left(a_{3}, i a_{2},-i a_{1}, a_{0}\right),
\end{aligned}
$$

que resulta em

$$
a_{0}=-i a_{2}, a_{1}=a_{3} \text { e } a_{0}=i a_{2}, a_{1}=a_{3},
$$

portanto

$$
a_{0}=a_{2}=0, a_{1}=a_{3} .
$$

Para satisfazer a Eq. (68) temos

$$
2\left|a_{1}\right|^{2}=1 \Rightarrow a_{1}=a_{3}=\frac{e^{i \varphi}}{\sqrt{2}}
$$

Impondo $\varphi=0$, obtemos

$$
U_{34}=\frac{1}{\sqrt{2}}(0,1,0,1)=\frac{1}{\sqrt{2}}\left(\begin{array}{cc}
1 & 1 \\
1 & -1
\end{array}\right) .
$$

Notamos que

$$
U_{34}=U_{34}^{\dagger}=U_{43}
$$

Para determinação da transformação $U_{15}: u_{p}^{[5]} \rightarrow$ $u_{p}^{[1]}$ da Eq. 73 e da Tabela (1) temos:

$$
U_{15}: \sigma^{1} U=U \sigma^{2}, \sigma^{2} U=U \sigma^{1} .
$$

Com o uso das Eqs. (70), temos

$$
\begin{aligned}
& \left(a_{1}, a_{0},-i a_{3}, i a_{2}\right)=\left(a_{2},-i a_{3}, a_{0}, i a_{1}\right), \\
& \left(a_{2}, i a_{3}, a_{0},-i a_{1}\right)=\left(a_{1}, a_{0}, i a_{3},-i a_{2}\right),
\end{aligned}
$$

que resulta em

$$
a_{0}=-i a_{3}, a_{1}=a_{2} \text { e } a_{0}=i a_{3}, a_{1}=a_{2},
$$

portanto

$$
a_{0}=a_{3}=0, a_{1}=a_{2} .
$$

Para satisfazer a Eq. 68 temos

$$
2\left|a_{1}\right|^{2}=1 \Rightarrow a_{1}=a_{2}=\frac{e^{i \varphi}}{\sqrt{2}}
$$

Impondo $\varphi=0$, obtemos

$$
U_{15}=\frac{1}{\sqrt{2}}(0,1,1,0)=\frac{1}{\sqrt{2}}\left(\begin{array}{cc}
0 & 1-i \\
1+i & 0
\end{array}\right) .
$$

Notamos que

$$
U_{15}=U_{15}^{\dagger}=U_{51}
$$

Agora determinamos a transformação $U_{24}: u_{p}^{[4]} \rightarrow$ $u_{p}^{[2]}$. Da Eq. (73) e da Tabela (1) temos:

$$
U_{24}: \sigma^{2} U=U \sigma^{1}, \sigma^{3} U=U \sigma^{3}
$$

Com o uso das Eqs. (70), temos

$$
\begin{aligned}
& \left(a_{2}, i a_{3}, a_{0},-i a_{1}\right)=\left(a_{1}, a_{0}, i a_{3},-i a_{2}\right), \\
& \left(a_{3},-i a_{2}, i a_{1}, a_{0}\right)=\left(a_{3}, i a_{2},-i a_{1}, a_{0}\right),
\end{aligned}
$$

que resulta em

$$
a_{2}=a_{1}, i a_{3}=a_{0} \text { e } a_{1}=0, a_{2}=0,
$$

portanto

$$
a_{1}=a_{2}=0, a_{3}=-i a_{0} .
$$

Para satisfazer a Eq. 68 temos

$$
2\left|a_{0}\right|^{2}=1 \Rightarrow a_{0}=\frac{e^{i \varphi}}{\sqrt{2}} \Rightarrow a_{3}=-i \frac{e^{i \varphi}}{\sqrt{2}},
$$

portanto

$$
U_{24}=\frac{e^{i \varphi}}{\sqrt{2}}(1,0,0,-i)=\frac{e^{i \varphi}}{\sqrt{2}}\left(\begin{array}{cc}
1-i & 0 \\
0 & 1+i
\end{array}\right) .
$$

Impondo $\varphi=0$, temos:

$$
U_{24}=\frac{1}{\sqrt{2}}\left(\begin{array}{cc}
1-i & 0 \\
0 & 1+i
\end{array}\right)=\frac{1}{\sqrt{2}}(1,0,0,-i) .
$$

A transformação inversa é determinada de acordo com a Eq. 64):

$$
U_{42}=U_{24}^{\dagger} \text {. }
$$

Observamos que as equações 96 escritas como

$$
\sigma^{3} U=U \sigma^{3}, \sigma^{2} U=U \sigma^{1},
$$

podem ser interpretadas como as relações para a determinação da transformação $U_{63}: u_{p}^{[3]} \rightarrow u_{p}^{[6]}$, portanto

$$
U_{63}=U_{36}^{\dagger}=U_{24}, U_{36}=U_{63}^{\dagger}=U_{24}^{\dagger}=U_{42} .
$$


Para a determinação da transformação $U_{16}$ $u_{p}^{[6]} \rightarrow u_{p}^{[1]}$ da Eq. 73 e da Tabela (1) temos:

$$
U_{16}: \sigma^{1} U=U \sigma^{3}, \sigma^{2} U=U \sigma^{2} .
$$

Com o uso das Eqs. (70), temos

$$
\begin{aligned}
& \left(a_{1}, a_{0},-i a_{3}, i a_{2}\right)=\left(a_{3}, i a_{2},-i a_{1}, a_{0}\right), \\
& \left(a_{2}, i a_{3}, a_{0},-i a_{1}\right)=\left(a_{2},-i a_{3}, a_{0}, i a_{1}\right),
\end{aligned}
$$

que resulta em

$$
a_{1}=a_{3}, a_{0}=i a_{2} \text { e } a_{1}=0, a_{3}=0,
$$

portanto

$$
a_{1}=a_{3}=0, a_{2}=-i a_{0} .
$$

Para satisfazer a Eq. 68 temos

$$
2\left|a_{0}\right|^{2}=1 \Rightarrow a_{0}=\frac{e^{i \varphi}}{\sqrt{2}} \Rightarrow a_{2}=-i \frac{e^{i \varphi}}{\sqrt{2}} .
$$

Impondo $\varphi=0$, obtemos

$$
U_{16}=\frac{1}{\sqrt{2}}(1,0,-i, 0)=\frac{1}{\sqrt{2}}\left(\begin{array}{cc}
1 & -1 \\
1 & 1
\end{array}\right) .
$$

A transformação inversa é determinada de acordo com a Eq. (64):

$$
U_{61}=U_{16}^{\dagger} .
$$

Observamos que as equações (99) escritas como

$$
\sigma^{2} U=U \sigma^{2}, \sigma^{1} U=U \sigma^{3},
$$

podem ser interpretadas como as relações para a determinação da transformação $U_{52}: u_{p}^{[2]} \rightarrow u_{p}^{[5]}$, portanto

$$
U_{52}=U_{16}=U_{25}^{\dagger}, U_{25}=U_{52}^{\dagger}=U_{16}^{\dagger}=U_{61} .
$$

Para a determinação da transformação $U_{14}$ : $u_{p}^{[4]} \rightarrow u_{p}^{[1]}$ da Eq. 73 e da Tabela (1) temos:

$$
U_{14}: \sigma^{1} U=U \sigma^{1}, \sigma^{2} U=U \sigma^{3}
$$

Usando as Eqs. (70), temos

$$
\begin{aligned}
& \left(a_{1}, a_{0},-i a_{3}, i a_{2}\right)=\left(a_{1}, a_{0}, i a_{3},-i a_{2}\right), \\
& \left(a_{2}, i a_{3}, a_{0},-i a_{1}\right)=\left(a_{3}, i a_{2},-i a_{1}, a_{0}\right),
\end{aligned}
$$

que resulta em

$$
a_{2}=a_{3}=0 \text { e } a_{2}=a_{3}, i a_{3}=i a_{2},
$$

portanto

$$
a_{2}=a_{3}=0, a_{1}=i a_{0} .
$$

Para satisfazer a Eq. 68 temos

$$
2\left|a_{0}\right|^{2}=1 \Rightarrow a_{0}=\frac{e^{i \varphi}}{\sqrt{2}}, a_{1}=i \frac{e^{i \varphi}}{\sqrt{2}} .
$$

Impondo $\varphi=0$, obtemos

$$
U_{14}=\frac{1}{\sqrt{2}}(1, i, 0,0)=\frac{1}{\sqrt{2}}\left(\begin{array}{ll}
1 & i \\
i & 1
\end{array}\right) .
$$

A transformação inversa é determinada de acordo com a Eq. 64):

$$
U_{41}=U_{14}^{\dagger} .
$$

Observamos que as equações 99 escritas como

$$
\sigma^{2} U=U \sigma^{3}, \sigma^{1} U=U \sigma^{1}
$$

podem ser interpretadas como as relações para a determinação da transformação $U_{53}: u_{p}^{[3]} \rightarrow u_{p}^{[5]}$, portanto

$$
U_{53}=U_{14}=U_{35}^{\dagger}, U_{35}=U_{53}^{\dagger}=U_{14}^{\dagger}=U_{41} .
$$

Todas as transformações entre representações cíclicas e anticíclicas são apresentadas nas Tabelas (3) e (4) na forma matricial, e na Tabela (5) na forma de componentes. Apresentamos também as propriedades das transformações:

$$
\begin{aligned}
& U_{34}=U_{43}, U_{26}=U_{62}, U_{15}=U_{51}, \\
& U_{16}=U_{25}^{\dagger}, U_{24}=U_{36}^{\dagger}, U_{14}=U_{35}^{\dagger} .
\end{aligned}
$$

\subsection{Transformações entre Representações dos Quartetos}

Nesta seção consideraremos as transformações entre uma representação principal e as representações não principais dentro de um quarteto, as transformações do tipo $U_{i_{0} i_{q}}$. Usaremos as Eqs. (57) na forma:

$$
\beta^{\left[i_{0}\right]} U_{i_{0} i_{q}}=U_{i_{0} i_{q}} \beta^{\left[i_{q}\right]}, \alpha^{\left[i_{0}\right]} U_{i_{0} i_{q}}=U_{i_{0} i_{q}} \alpha^{\left[i_{q}\right]} .
$$

Determinaremos as transformações com $i=1$, isto é, $U_{1_{0} 1_{q}}$. Para $q=1$, da Eq. 107) e da Tabela (1) temos:

$$
q=1: \sigma^{1} U=U \sigma^{1},-\sigma^{2} U=U \sigma^{2}
$$

$\mathrm{Ou}$

$\left[\sigma^{1}, U\right]=\sigma^{1} U-U \sigma^{1}=0,\left\{\sigma^{2}, U\right\}=\sigma^{2} U+U \sigma^{2}=0$. 
Tabela 3: As transformações $U_{i j}$ das representações anticíclicas para as representações cíclicas na forma matricial.

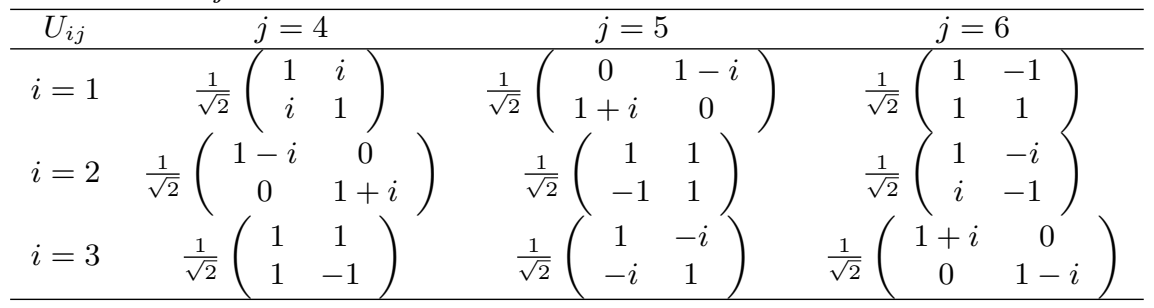

Tabela 4: As transformações $U_{j i}$ das representações cíclicas para as representações anticíclicas na forma matricial.

\begin{tabular}{cccc}
\hline$U_{j i}$ & $i=1$ & $i=2$ & $i=3$ \\
\hline$j=4$ & $\frac{1}{\sqrt{2}}\left(\begin{array}{cc}1 & -i \\
-i & 1\end{array}\right)$ & $\frac{1}{\sqrt{2}}\left(\begin{array}{cc}1+i & 0 \\
0 & 1-i\end{array}\right)$ & $\frac{1}{\sqrt{2}}\left(\begin{array}{cc}1 & 1 \\
1 & -1\end{array}\right)$ \\
$j=5$ & $\frac{1}{\sqrt{2}}\left(\begin{array}{cc}0 & 1-i \\
1+i & 0\end{array}\right)$ & $\frac{1}{\sqrt{2}}\left(\begin{array}{cc}1 & -1 \\
1 & 1 \\
1 & -i \\
i & -1\end{array}\right)$ & $\frac{1}{\sqrt{2}}\left(\begin{array}{cc}1 & i \\
i & 1\end{array}\right)$ \\
$j=6$ & $\frac{1}{\sqrt{2}}\left(\begin{array}{cc}1 & 1 \\
-1 & 1\end{array}\right)$ & $\frac{1}{\sqrt{2}}\left(\begin{array}{cc}1-i & 0 \\
0 & 1+i\end{array}\right)$ \\
\hline
\end{tabular}

Tabela 5: As transformações entre representações cíclicas e anticíclicas $U_{i j}, U_{j i}$ na forma de componentes.

\begin{tabular}{cccc}
\hline$U_{i j}$ & $j=4$ & $j=5$ & $j=6$ \\
\hline$i=1$ & $\frac{1}{\sqrt{2}}(1, i, 0,0)$ & $\frac{1}{\sqrt{2}}(0,1,1,0)$ & $\frac{1}{\sqrt{2}}(1,0,-i, 0)$ \\
$i=2$ & $\frac{1}{\sqrt{2}}(1,0,0,-i)$ & $\frac{1}{\sqrt{2}}(1,0, i, 0)$ & $\frac{1}{\sqrt{2}}(0,0,1,1)$ \\
$i=3$ & $\frac{1}{\sqrt{2}}(0,1,0,1)$ & $\frac{1}{\sqrt{2}}(1,-i, 0,0)$ & $\frac{1}{\sqrt{2}}(1,0,0, i)$ \\
\hline \multicolumn{4}{c}{} \\
\hline$U_{j i}$ & $i=1$ & $i=2$ & $i=3$ \\
\hline$j=4$ & $\frac{1}{\sqrt{2}}(1,-i, 0,0)$ & $\frac{1}{\sqrt{2}}(1,0,0, i)$ & $\frac{1}{\sqrt{2}}(0,1,0,1)$ \\
$j=5$ & $\frac{1}{\sqrt{2}}(0,1,1,0)$ & $\frac{1}{\sqrt{2}}(1,0,-i, 0)$ & $\frac{1}{\sqrt{2}}(1, i, 0,0)$ \\
$j=6$ & $\frac{1}{\sqrt{2}}(1,0, i, 0)$ & $\frac{1}{\sqrt{2}}(0,0,1,1)$ & $\frac{1}{\sqrt{2}}(1,0,0,-i)$ \\
\hline
\end{tabular}

Com o uso das Eqs. (71) e (72) obtemos

$$
a_{2}=a_{3}=0 ; a_{0}=a_{2}=0
$$

que dá

$$
a_{0}=a_{2}=a_{3}=0 \text {. }
$$

Para satisfazer a Eq. (68) temos:

$$
\left|a_{1}\right|=1 \Rightarrow a_{1}=e^{i \varphi} \text {. }
$$

Escolhendo $\varphi=0$, obtemos

$$
U_{1_{0} 1_{1}}=(0,1,0,0)=\sigma^{1} .
$$

Para $q=2$, da Eq. (107) e da Tabela (1) temos:

$$
q=2:-\sigma^{1} U=U \sigma^{1}, \sigma^{2} U=U \sigma^{2}
$$

ou

$$
\left\{\sigma^{1}, U\right\}=0,\left[\sigma^{2}, U\right]=0 .
$$

Com o uso das Eqs. 71 e 72 obtemos

$$
a_{0}=a_{1}=0 ; a_{1}=a_{3}=0 \text {, }
$$

que dá

$$
a_{0}=a_{1}=a_{3}=0 .
$$

Para satisfazer a Eq. (68) temos:

$$
\left|a_{2}\right|=1 \Rightarrow a_{2}=e^{i \varphi} \text {. }
$$

Escolhendo $\varphi=0$, obtemos

$$
U_{1_{0} 1_{2}}=(0,0,1,0)=\sigma^{2} .
$$

Para $q=3$, da Eq. (107) e da Tabela (1) temos:

$$
q=3:-\sigma^{1} U=U \sigma^{1},-\sigma^{2} U=U \sigma^{2}
$$

$\mathrm{Ou}$

$$
\left\{\sigma^{1}, U\right\}=0,\left\{\sigma^{2}, U\right\}=0 .
$$

Usando as Eqs. 71 e 72 obtemos

$$
a_{0}=a_{1}=0 ; a_{0}=a_{2}=0,
$$

que dá

$$
a_{0}=a_{1}=a_{2}=0 .
$$

Para satisfazer a Eq. 68 temos

$$
\left|a_{3}\right|=1 \Rightarrow a_{3}=e^{i \varphi} \text {. }
$$


Escolhendo $\varphi=0$, obtemos

$$
U_{1_{0} 1_{3}}=(0,0,0,1)=\sigma^{3} .
$$

As Eqs. 108 escritas como

$$
-\sigma^{2} U=U \sigma^{2}, \sigma^{1} U=U \sigma^{1}: q=2
$$

podem ser interpretadas como as relações para determinação da transformação $U_{5_{0} 5_{2}}$ do setor anticíclico, portanto

$$
U_{5_{0} 5_{2}}=U_{1_{0} 1_{1}}=\sigma^{1} .
$$

As Eqs. 110 escritas como

$$
\sigma^{2} U=U \sigma^{2},-\sigma^{1} U=U \sigma^{1}: q=1
$$

podem ser interpretadas como as relações para determinação da transformação $U_{5_{0} 5_{1}}$ do setor anticíclico, portanto

$$
U_{5_{0} 5_{1}}=U_{1_{0} 1_{2}}=\sigma^{2} .
$$

As Eqs. 112) escritas como

$$
-\sigma^{2} U=U \sigma^{2},-\sigma^{1} U=U \sigma^{1}: q=3
$$

podem ser interpretadas como as relações para determinação da transformação $U_{5_{0} 5_{3}}$ do setor anticíclico, portanto

$$
U_{5_{0} 5_{3}}=U_{1_{0} 1_{3}}=\sigma^{3} .
$$

Para determinar as transformações com $i=2,3$ do setor cíclico e as correspondentes transformações com $i=6,4$ do setor anticíclico procedemos de forma semelhante e obtemos os resultados:

$$
\begin{aligned}
& U_{2_{0} 2_{1}}=U_{6_{0} 6_{2}}=\sigma^{2}, U_{2_{0} 2_{2}}=U_{6_{0} 6_{1}}=\sigma^{3}, \\
& U_{2_{0} 2_{3}}=U_{6_{0} 6_{3}}=\sigma^{1} . \\
& U_{3_{0} 3_{1}}=U_{4_{0} 4_{2}}=\sigma^{3}, U_{3_{0} 3_{2}}=U_{4_{0} 4_{1}}=\sigma^{1}, \\
& U_{3_{0} 3_{3}}=U_{4_{0} 4_{3}}=\sigma^{2} .
\end{aligned}
$$

Os resultados dos cálculos das transformações do tipo $U_{i_{0} i_{q}}$ são apresentados na Tabela (6).

Observamos que

$$
U_{i_{0}, i_{q}}=U_{i_{q}, i_{0}} .
$$

\section{Estrutura Algébrica do Conjunto das Transformações Unitárias}

Nesta seção estudaremos a estrutura algébrica do conjunto das transformações unitárias entre as várias representações. Antes disso, daremos uma noção de grupos como entidade matemática para os leitores que não tem conhecimento neste campo. Textos introdutórios sobre este assunto podem ser encontrados, por exemplo, nas Refs. 21] e 22.

\subsection{Definições Básicas da Teoria de Grupos}

Um conjunto de elementos $G$ em que é definida uma operação $(\times)$, entre dois elementos, forma um grupo, se, para seus elementos é satisfeito o seguinte sistema de axiomas:

1. Sejam $g_{1}$ e $g_{2}$ elementos de $G$, então o elemento $g_{1} \times g_{2}$ também pertence a $G$ :

$$
\forall g_{1}, g_{2} \in G, g_{1} \times g_{2} \in G \text {. }
$$

2. Para todos os elementos pertencentes a $G$, é satisfeita a lei de associatividade, isto é, para quaisquer três elementos $g_{1}, g_{2}$ e $g_{3}$ pertencentes a $G$, é satisfeita a relação:

$$
\forall g_{1}, g_{2}, g_{3} \in G,\left(g_{1} \times g_{2}\right) \times g_{3}=g_{1} \times\left(g_{2} \times g_{3}\right) .
$$

3. Existe o elemento neutro $e$ em $G$ :

$$
\exists e \in G: \forall g \in G, e \times g=g \times e=g .
$$

O elemento neutro $e$ é único.

4. Para cada elemento $g$ em $G$ existe um elemento inverso $g^{-1}$ que também pertence a $G$ :

$$
\forall g \in G, \exists g^{-1}: g \times g^{-1}=g^{-1} \times g=e .
$$

O elemento inverso $g^{-1}$ é único.

Tabela 6: As transformações $U_{i_{0}, i_{q}}$ entre uma representação principal e as representações não principais para os quartetos indicados por $i$.

\begin{tabular}{cccc}
\hline$U_{i_{0}, i_{q}}$ & $q=1(+-)$ & $q=2(-+)$ & $q=3(--)$ \\
\hline$i=1$ & $\sigma^{1}=(0,1,0,0)$ & $\sigma^{2}=(0,0,1,0)$ & $\sigma^{3}=(0,0,0,1)$ \\
$i=2$ & $\sigma^{2}$ & $\sigma^{3}$ & $\sigma^{1}$ \\
$i=3$ & $\sigma^{3}$ & $\sigma^{1}$ & $\sigma^{2}$ \\
$i=4$ & $\sigma^{1}$ & $\sigma^{3}$ & $\sigma^{2}$ \\
$i=5$ & $\sigma^{2}$ & $\sigma^{1}$ & $\sigma^{3}$ \\
$i=6$ & $\sigma^{3}$ & $\sigma^{2}$ & $\sigma^{1}$ \\
\hline
\end{tabular}


O axioma 1 é denominado de axioma de fechamento, o $\mathbf{2}$ de associatividade, o $\mathbf{3}$ de elemento neutro e o 4 de elemento inverso.

Se para os quatro axiomas acima é adicionado um axioma de comutatividade:

5. Para quaisquer elementos $g_{1}$ e $g_{2}$ pertencentes a $G$, é satisfeita a relação:

$$
\forall g_{1}, g_{2} \in G, g_{1} \times g_{2}=g_{2} \times g_{1},
$$

o grupo é denominado de grupo comutativo ou abeliano. Se um grupo não obedece o axioma de comutatividade, ele é denominado de grupo não comutativo ou não abeliano.

Se um subconjunto $G^{\prime}$ de $G$ é fechado sob a operação $(\times)$, isto é, para todos elementos $g_{1}^{\prime} \mathrm{e}$ $g_{2}^{\prime}$ de $G^{\prime}$ temos que $g_{1}^{\prime} \times g_{2}^{\prime}$ também pertence a $G^{\prime}$ :

$$
\forall g_{1}^{\prime}, g_{2}^{\prime} \in G^{\prime}, g_{1}^{\prime} \times g_{2}^{\prime} \in G^{\prime},
$$

então, $G^{\prime}$ forma um grupo e é denominado subgrupo de $G$.

Apresentamos alguns exemplos simples de grupos.

1. O conjunto de números inteiros $\mathbb{Z}$ com a operação de soma, que designamos por $(\mathbb{Z},+)$. É simples verificar os axiomas acima. A soma de quaisquer dois números inteiros é um número inteiro. A operação de soma para números inteiros é associativa. $\mathrm{O}$ elemento neutro em $\mathbb{Z}$ é o zero, 0 . Para qualquer número inteiro $n$ o elemento inverso é $-n$. Então, $(\mathbb{Z},+)$ é um grupo. A soma de dois números inteiros é comutativa, isto é, o resultado não depende da ordem dos números somados. Portanto, o grupo $(\mathbb{Z},+)$ é abeliano.

2. O conjunto de números reais $\mathbb{R}$ com a operação de soma, que designamos por $(\mathbb{R},+)$. A soma de quaisquer dois números reais é um número real. A operação de soma para números reais é associativa. O elemento neutro em $(\mathbb{R},+)$ é o zero, 0 . Para qualquer número real $x$ o elemento inverso em $(\mathbb{R},+)$ é $-x$. Então, $(\mathbb{R},+)$ forma um grupo. Por razões similares ao exemplo 1 , podemos concluir que $(\mathbb{R},+)$ é um grupo abeliano.

3. O conjunto de números reais positivos $\mathbb{R}_{+}$com a operação de multiplicação, que designamos por $\left(\mathbb{R}_{+}, \cdot\right)$. O produto de quaisquer dois números reais positivos é um número real positivo. A operação de multiplicação para números reais positivos é associativa. O elemento neutro em $\left(\mathbb{R}_{+}, \cdot\right)$ é a unidade, 1. Para qualquer número real $x$ o elemento inverso em $\left(\mathbb{R}_{+}, \cdot\right)$ é $1 / x$. Então, $\left(\mathbb{R}_{+}, \cdot\right)$ forma um grupo. O produto de números reais positivos é comutativo, isto é, o resultado não depende da ordem dos números multiplicados. Portanto o grupo $\left(\mathbb{R}_{+}, \cdot\right)$ é abeliano.

Agora apresentamos alguns exemplos de subgrupos.

1. Nos exemplos 1 e 2 acima mostramos que os conjuntos $\mathbb{Z}$ e $\mathbb{R}$ com a operação de soma formam grupos. $\mathbb{Z}$ é o subconjunto de $\mathbb{R}$, portanto $\mathbb{Z}$ é um subgrupo de $\mathbb{R}$.

2. Consideremos o conjunto de todos os números ímpares com a operação de soma que é um subconjunto de números inteiros. Este conjunto não forma um grupo, pois a soma de dois números ímpares é um número par. Portanto, o axioma de fechamento não é obedecido. Entretanto, a situação é outra com o conjunto de todos os números pares, que também é um subconjunto dos números inteiros. Ele obedece os axiomas de grupo: a soma de dois números pares é um número par, a operação de soma de números pares é associativa, o elemento neutro é o zero, o elemento inverso para um dado número par é o número com o sinal oposto. Portanto, o conjunto de todos números pares é um subgrupo de números inteiros.

\subsection{Estrutura Algébrica do Conjunto das Transformações}

O conjunto que consideraremos nesta seção é o conjunto das transformações unitárias entre as várias representações das matrizes de Dirac que foi obtido nas seções anteriores. Cada transformação é representada por uma matriz unitária. Uma ação consecutiva das transformações, que introduz uma composição de transformações, é definida como o produto usual de matrizes. Nosso objeto de estudo é o conjunto de matrizes unitárias indicado na Eq. 65):

$$
U_{j_{r} i_{q}}=U_{j_{r} j_{0}} U_{j_{0} i_{0}} U_{i_{0} i_{q}} .
$$

Entre os elementos do conjunto é definida uma operação de multiplicação: o produto matricial. O produto de matrizes é uma operação associativa. Sendo $U$ transformações unitárias, as transformações inversas são definidas por $U^{-1}=U^{\dagger}$. Ressaltamos que entre as matrizes na Eq. (126) está presente também a matriz unidade.

$\mathrm{Na}$ investigação da estrutura do conjunto não distinguiremos as transformações que diferem por um fator de fase. Em outras palavras, diremos que as transformações que diferem por um fator de fase pertencem a uma mesma classe de equivalência, que 
denotamos pelo símbolo $\simeq$

$$
U \simeq e^{i \varphi} U .
$$

Primeiramente, observamos que o conjunto que inclui as matrizes de Pauli $\sigma^{i}$ e a matriz unidade $\sigma^{0}$, representado por $\sigma^{\mu}=\left\{\sigma^{0}, \sigma^{i}\right\}$, forma um grupo em classes de equivalência, porque

$$
\sigma^{\mu} \sigma^{\nu} \simeq \sigma^{\lambda}
$$

O conjunto de três transformações $\left\{\sigma^{0}, U_{0}, U_{0}^{\dagger}\right\}$ também forma um grupo em classes de equivalência, porque

$$
U_{0}^{2} \simeq U_{0}^{\dagger}, U_{0}^{\dagger 2} \simeq U_{0},
$$

que foi indicado na Eq. 86 .

\subsubsection{Estrutura do Setor Cíclico (Anticíclico) das Transformações}

Na primeira etapa discutiremos as transformações entre as representações do setor cíclico (anticíclico). Qualquer transformação, inclusive as transformações inversas, no setor cíclico (anticíclico) pode ser apresentada em uma das formas:

$$
\sigma^{\mu} \sigma^{0} \sigma^{\nu}, \sigma^{\mu} U_{0} \sigma^{\nu}, \sigma^{\mu} U_{0}^{\dagger} \sigma^{\nu} .
$$

Levando em conta a Eq. (127), temos:

$$
\sigma^{\mu} \sigma^{0} \sigma^{\nu} \simeq \sigma^{\mu} \sigma^{\nu} \simeq \sigma^{\lambda} .
$$

Portanto, o conjunto das transformações do setor cíclico (anticíclico), que denotamos por $\mathbf{X}$, tem a forma:

$$
\mathbf{X}=\left\{\sigma^{\lambda}, \sigma^{\mu} U_{0} \sigma^{\nu}, \sigma^{\alpha} U_{0}^{\dagger} \sigma^{\beta}\right\} .
$$

Além disso, por cálculos diretos pode ser demonstrado que

$$
\sigma^{1} U_{0} \simeq U_{0} \sigma^{3}, \sigma^{2} U_{0} \simeq U_{0} \sigma^{1}, \sigma^{3} U_{0} \simeq U_{0} \sigma^{2}
$$

e

$$
U_{0}^{\dagger} \sigma^{1} \simeq \sigma^{3} U_{0}^{\dagger}, U_{0}^{\dagger} \sigma^{2} \simeq \sigma^{1} U_{0}^{\dagger}, U_{0}^{\dagger} \sigma^{3} \simeq \sigma^{2} U_{0}^{\dagger},
$$

que expressamos de forma simbólica como:

$$
\sigma^{\mu} U_{0} \simeq U_{0} \sigma^{\nu}, \sigma^{\mu} U_{0}^{\dagger} \simeq U_{0}^{\dagger} \sigma^{\nu} .
$$

Denotaremos os subconjuntos do conjunto $\mathbf{X}$ por:

$$
C=\left\{\sigma^{\lambda}\right\}, A=\left\{\sigma^{\mu} U_{0} \sigma^{\nu}\right\}, B=\left\{\sigma^{\alpha} U_{0}^{\dagger} \sigma^{\beta}\right\} .
$$

Usando a Eq. (127), temos:

$$
\begin{aligned}
& \forall c_{1}, c_{2} \in C, c_{1} c_{2}=\sigma^{\mu} \sigma^{\nu} \simeq \sigma^{\lambda} \in C, \\
& \forall c \in C, \forall a \in A, c a=\sigma^{\lambda} \sigma^{\mu} U_{0} \sigma^{\nu} \\
& \simeq \sigma^{\mu^{\prime}} U_{0} \sigma^{\nu} \in A \\
& a c=\sigma^{\mu} U_{0} \sigma^{\nu} \sigma^{\lambda} \simeq \sigma^{\mu} U_{0} \sigma^{\nu^{\prime}} \in A \text {, } \\
& \forall c \in C, \forall b \in B, c b=\sigma^{\lambda} \sigma^{\mu} U_{0}^{\dagger} \sigma^{\nu} \\
& \simeq \sigma^{\mu^{\prime}} U_{0}^{\dagger} \sigma^{\nu} \in B \\
& b c=\sigma^{\mu} U_{0}^{\dagger} \sigma^{\nu} \sigma^{\lambda} \simeq \sigma^{\mu} U_{0}^{\dagger} \sigma^{\nu^{\prime}} \in B .
\end{aligned}
$$

Usando as Eqs. 127, 130 e 131, temos:

$$
\begin{aligned}
\forall a \in & A, \forall b \in B, a b=\sigma^{\mu} U_{0} \sigma^{\nu} \sigma^{\alpha} U_{0}^{\dagger} \sigma^{\beta} \\
& \simeq \sigma^{\mu} \sigma^{\nu^{\prime}} U_{0} U_{0}^{\dagger} \sigma^{\alpha^{\prime}} \sigma^{\beta} \simeq \sigma^{\mu^{\prime}} \sigma^{\beta^{\prime}} \simeq \sigma^{\lambda} \in C ; \\
b a= & \sigma^{\alpha} U_{0}^{\dagger} \sigma^{\beta} \sigma^{\mu} U_{0} \sigma^{\nu} \simeq \sigma^{\alpha} \sigma^{\beta^{\prime}} U_{0}^{\dagger} U_{0} \sigma^{\mu^{\prime}} \sigma^{\nu} \\
& \simeq \sigma^{\alpha^{\prime}} \sigma^{\nu^{\prime}} \simeq \sigma^{\lambda} \in C .
\end{aligned}
$$

Usando as Eqs. (127), 130, 131) e a Eq. 128, temos:

$$
\begin{aligned}
\forall a_{1}, a_{2} \in & A, a_{1} a_{2}=\sigma^{\mu} U_{0} \sigma^{\nu} \sigma^{\alpha} U_{0} \sigma^{\beta} \\
& \simeq \sigma^{\mu} \sigma^{\nu^{\prime}} U_{0}^{2} \sigma^{\alpha^{\prime}} \sigma^{\beta} \simeq \sigma^{\mu^{\prime}} U_{0}^{\dagger} \sigma^{\beta^{\prime}} \in B ; \\
\forall b_{1}, b_{2} \in & B, b_{1} b_{2}=\sigma^{\mu} U_{0}^{\dagger} \sigma^{\nu} \sigma^{\alpha} U_{0}^{\dagger} \sigma^{\beta} \\
& \simeq \sigma^{\mu} \sigma^{\nu^{\prime}} U_{0}^{\dagger 2} \sigma^{\alpha^{\prime}} \sigma^{\beta} \simeq \sigma^{\mu^{\prime}} U_{0} \sigma^{\beta^{\prime}} \in A .
\end{aligned}
$$

Então, o conjunto $\mathbf{X}$ contém o elemento neutro e possui as propriedades:

$$
\forall x \in X, x^{-1} \in \mathbf{X} ; \forall x_{1}, x_{2} \in X, x_{1} x_{2} \in \mathbf{X},
$$

que significa que o conjunto $\mathbf{X}$ forma um grupo. $\mathrm{O}$ grupo $\mathbf{X}$ possui a seguinte subestrutura:

$$
\begin{aligned}
\forall a_{1}, a_{2} & \in A, a_{1} a_{2} \in B ; \\
\forall b_{1}, b_{2} & \in B, b_{1} b_{2} \in A ; \\
\forall c_{1}, c_{2} & \in C, c_{1} c_{2} \in C ; \\
\forall a & \in A, \forall b \in B, a b \in C, b a \in C ; \\
\forall a & \in A, \forall c \in C, a c \in A, c a \in A ; \\
\forall b & \in B, \forall c \in C, b c \in B, c b \in B .
\end{aligned}
$$

As propriedades de multiplicação dos elementos dos subconjuntos do grupo $\mathbf{X}$ são apresentadas também na Tabela (7) e na Fig. (11). Das propriedades (130) e (131), concluímos que o grupo $\mathbf{X}$ é não abeliano. Além disso, de acordo com as Eqs. (127) e (128), o grupo $\mathbf{X}$ contém dois subgrupos não triviais:

$$
G_{U}=\left\{\sigma^{0}, U_{0}, U_{0}^{\dagger}\right\}, G_{C}=\left\{\sigma^{\mu}\right\} .
$$


Tabela 7: Tabela de efeito de multiplicação dos elementos dos subconjuntos $A, B, C$ do grupo $\mathbf{X}$.

\begin{tabular}{c|ccc}
\hline $\mathrm{X}$ & $\mathrm{C}$ & $\mathrm{A}$ & $\mathrm{B}$ \\
\hline $\mathrm{C}$ & $\mathrm{C}$ & $\mathrm{A}$ & $\mathrm{B}$ \\
$\mathrm{A}$ & $\mathrm{A}$ & $\mathrm{B}$ & $\mathrm{C}$ \\
$\mathrm{B}$ & $\mathrm{B}$ & $\mathrm{C}$ & $\mathrm{A}$ \\
\hline
\end{tabular}

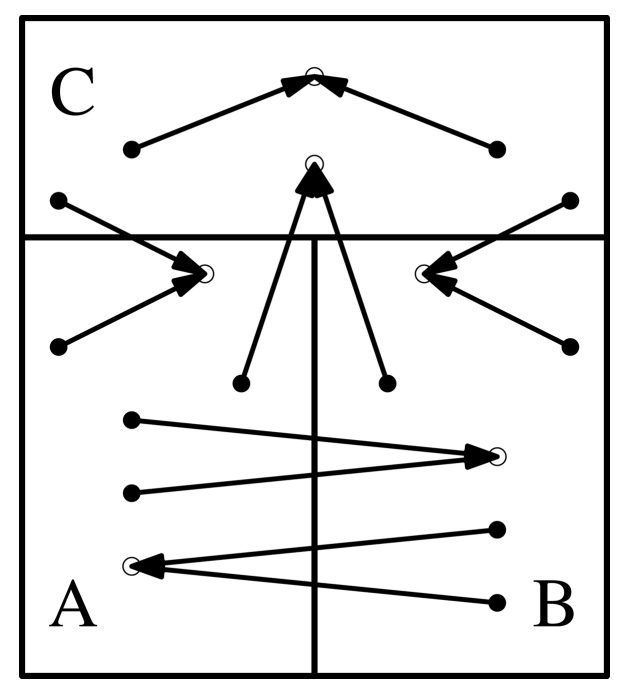

Figura 1: Esquema da estrutura do grupo $\mathbf{X}$.

\subsubsection{Estrutura do Conjunto Total das Transformações}

Agora discutiremos o conjunto total das transformações. O conjunto total inclui o conjunto das transformações entre as representações do setor cíclico (anticíclico) $\mathbf{X}$, estudado na seção anterior, e um conjunto das transformações entre representações cíclicas e anticíclicas, que denotaremos por Y. Notamos que as transformações entre as representações cíclicas e anticíclicas principais, que são apresentadas nas Tabelas (3), (4), podem ser escritas na forma:

$$
\begin{gathered}
U_{t}, U_{t} U_{0}, U_{0} U_{t}, U_{t} U_{0}^{\dagger}, U_{0}^{\dagger} U_{t}, \\
U_{0} U_{t} U_{0}, U_{0} U_{t} U_{0}^{\dagger}, U_{0}^{\dagger} U_{t} U_{0}, U_{0}^{\dagger} U_{t} U_{0}^{\dagger},
\end{gathered}
$$

onde $U_{0}, U_{0}^{\dagger}$ são definidas nas Eqs. 84 e 85 , e $U_{t}$ é uma transformação de transição entre as representações cíclicas e anticíclicas. Para $U_{t}$ é conveniente escolher a transformação $U_{26}$, pois $U_{26}=$ $U_{26}^{\dagger}=U_{62}$ :

$$
U_{t}=U_{26}, U_{t}=U_{t}^{\dagger} \Rightarrow U_{t}^{2}=1
$$

Escrevemos cada uma das transformações da Tabela (3) na forma de composição das transformações apropriadas utilizando as Eqs. 84, 85) e (141):

$$
\begin{aligned}
& U_{14}=U_{12} U_{26} U_{64} \simeq U_{0}^{\dagger} U_{t} U_{0}^{\dagger}, U_{15}=U_{12} U_{26} U_{65} \simeq U_{0}^{\dagger} U_{t} U_{0}, U_{16}=U_{12} U_{26} \simeq U_{0}^{\dagger} U_{t}, \\
& U_{24}=U_{26} U_{64} \simeq U_{t} U_{0}^{\dagger}, U_{25}=U_{26} U_{65} \simeq U_{t} U_{0}, U_{26} \simeq U_{t} \\
& U_{34}=U_{32} U_{26} U_{64} \simeq U_{0} U_{t} U_{0}^{\dagger}, U_{35}=U_{32} U_{26} U_{65} \simeq U_{0} U_{t} U_{0}, U_{36}=U_{32} U_{26} \simeq U_{0} U_{t}
\end{aligned}
$$

Denotamos os elementos do grupo $G_{U}=$ $\left\{\sigma^{0}, U_{0}, U_{0}^{\dagger}\right\}$ por $V_{i}$ :

$$
\begin{aligned}
& V_{i} \in G_{U}, i=0, \pm 1: V_{0}=\sigma^{0} \\
& V_{+1}=U_{0}, V_{-1}=U_{0}^{\dagger} .
\end{aligned}
$$

Para $V_{i}$ temos:

$$
\forall V_{i}, V_{j} \in G_{U}, V_{i} V_{j} \simeq V_{k} \in G_{U} ; V_{i}^{\dagger} \simeq V_{j} .
$$

As transformações do conjunto (140) podem ser escritas na forma simbólica como:

$$
V_{i} U_{t} V_{j}
$$

Adicionando ainda as transformações não principais, podemos escrever o conjunto $\mathbf{Y}$ das transformações entre as representações cíclicas e anticíclicas na forma:

$$
\mathbf{Y}=\left\{\sigma^{\mu} V_{i} U_{t} V_{j} \sigma^{\nu}\right\} .
$$

Por cálculo direto, pode ser demonstrado que

$$
U_{t} U_{0} U_{t} \simeq U_{0}^{\dagger} \sigma^{3}, U_{t} U_{0}^{\dagger} U_{t} \simeq U_{0} \sigma^{2}
$$

$\mathrm{ou}$

$$
\begin{aligned}
& U_{0} U_{t} \simeq U_{t} U_{0}^{\dagger} \sigma^{3}, U_{0}^{\dagger} U_{t} \simeq U_{t} U_{0} \sigma^{2}, \\
& U_{0} U_{t} \sigma^{3} \simeq U_{t} U_{0}^{\dagger}, U_{0}^{\dagger} U_{t} \sigma^{2} \simeq U_{t} U_{0}
\end{aligned}
$$

que expressamos simbolicamente como:

$$
V_{i} U_{t} \simeq U_{t} V_{j} \sigma^{\mu}
$$


Por cálculo direto, pode ser demonstrado também que

$$
\sigma^{1} U_{t}=-U_{t} \sigma^{1}, \sigma^{2} U_{t}=U_{t} \sigma^{3}, \sigma^{3} U_{t}=U_{t} \sigma^{2},
$$

que, por sua vez, pode ser simbolicamente expressada como

$$
\sigma^{\mu} U_{t} \simeq U_{t} \sigma^{\nu}
$$

O conjunto total das transformações denotamos por:

$$
\Omega=\{\mathbf{X}, \mathbf{Y}\}=\left\{\sigma^{\alpha} V_{k} \sigma^{\beta}, \sigma^{\mu} V_{i} U_{t} V_{j} \sigma^{\nu}\right\} ; i, j, k=0, \pm 1 ; \alpha, \beta, \mu, \nu=0,1,2,3
$$

Usando as Eqs. 146, 148) e 149, temos:

$$
\begin{aligned}
& \forall y_{1}, y_{2} \in \mathbf{Y}, y_{1} y_{2}=\sigma^{\mu} V_{i} U_{t} V_{j} \sigma^{\nu} \sigma^{\alpha} V_{k} U_{t} V_{l} \sigma^{\beta} \simeq \sigma^{\mu} V_{i} U_{t} V_{j} \sigma^{\nu^{\prime}} V_{k} U_{t} V_{l} \sigma^{\beta} \\
& \simeq \sigma^{\mu} V_{i} U_{t} \sigma^{\alpha^{\prime}} V_{j} V_{k} U_{t} V_{l} \sigma^{\beta} \simeq \sigma^{\mu} V_{i} U_{t} \sigma^{\alpha^{\prime}} V_{k^{\prime}} U_{t} V_{l} \sigma^{\beta} \simeq \sigma^{\mu} V_{i} \sigma^{\lambda} U_{t}^{2} V_{j} \sigma^{\nu} V_{l} \sigma^{\beta} \\
& \simeq \sigma^{\mu} V_{i} \sigma^{\lambda} V_{j} \sigma^{\nu} V_{l} \sigma^{\beta} \simeq \sigma^{\mu} \sigma^{\lambda^{\prime}} V_{i} V_{j} V_{l} \sigma^{\nu^{\prime}} \sigma^{\beta} \simeq \sigma^{\mu^{\prime}} V_{j^{\prime}} V_{l} \sigma^{\beta^{\prime}} \simeq \sigma^{\mu^{\prime}} V_{l^{\prime}} \sigma^{\beta^{\prime}} \in \mathbf{X} . \\
& \forall x \quad \in \quad \mathbf{X}, \forall y \in \mathbf{Y}, x y=\sigma^{\mu} V_{i} \sigma^{\nu} \sigma^{\alpha} V_{k} U_{t} V_{l} \sigma^{\beta} \simeq \sigma^{\mu} V_{i} \sigma^{\alpha^{\prime}} U_{t} V_{k^{\prime}} \sigma^{\lambda} V_{l} \sigma^{\beta} \\
& \simeq \sigma^{\mu} \sigma^{\alpha^{\prime}} V_{i} U_{t} V_{k^{\prime}} V_{l} \sigma^{\lambda^{\prime}} \sigma^{\beta} \simeq \sigma^{\mu^{\prime}} V_{i} U_{t} V_{m} \sigma^{\beta^{\prime}} \in \mathbf{Y} ; \\
& y x=\sigma^{\alpha} V_{k} U_{t} V_{l} \sigma^{\beta} \sigma^{\mu} V_{i} \sigma^{\nu} \simeq \sigma^{\alpha} V_{k} U_{t} V_{l} \sigma^{\mu^{\prime}} V_{i} \sigma^{\nu} \\
& \simeq \sigma^{\alpha} V_{k} U_{t} V_{l} V_{i} \sigma^{\beta^{\prime}} \sigma^{\nu} \simeq \sigma^{\alpha} V_{k} U_{t} V_{m} \sigma^{\lambda} \in \mathbf{Y} ; \\
& \forall x_{1}, x_{2} \in X, x_{1} x_{2}=\sigma^{\mu} V_{i} \sigma^{\nu} \sigma^{\alpha} V_{j} \sigma^{\beta} \simeq \sigma^{\mu} V_{i} \sigma^{\nu^{\prime}} V_{j} \sigma^{\beta} \\
& \simeq \sigma^{\mu} \sigma^{\alpha^{\prime}} V_{i} V_{j} \sigma^{\beta} \simeq \sigma^{\mu^{\prime}} V_{k} \sigma^{\beta} \in \mathbf{X} .
\end{aligned}
$$

Então, o conjunto $\Omega$ contém um elemento neutro e possui as propriedades:

$$
\forall \omega \in \Omega, \omega^{-1} \in \Omega ; \forall \omega_{1}, \omega_{2} \in \Omega, \omega_{1} \omega_{2} \in \Omega,
$$

que significa que o conjunto $\Omega$ forma um grupo. $\mathrm{O}$ grupo $\Omega$ possui a subestrutura:

$$
\forall x \in \mathbf{X}, \forall y \in \mathbf{Y}, x y \in \mathbf{Y}, y x \in \mathbf{Y},
$$
$\forall y_{1}, y_{2} \in \mathbf{Y}, y_{1} y_{2} \in \mathbf{X} ; \forall x_{1}, x_{2} \in \mathbf{X}, x_{1} x_{2} \in \mathbf{X}$.

As propriedades de multiplicação dos elementos dos subconjuntos do grupo $\Omega$ são apresentadas também na Tabela (8) e na Fig. (2). Das propriedades (145), (147), 130 e (131), concluímos que o grupo $\Omega$ é não abeliano. Além disso, de acordo com a Eq. (156) e as Eqs. 127), 128 o grupo $\Omega$ contém um subgrupo não trivial:

$$
G_{X}=\left\{\sigma^{\mu} V_{i} \sigma^{\nu}\right\}
$$

que, por sua vez, contém os dois subgrupos não triviais:

$$
G_{U}=\left\{V_{i}\right\}, G_{C}=\left\{\sigma^{\mu}\right\}
$$

Tabela 8: Tabela de efeito de multiplicação dos elementos dos subconjuntos $\mathbf{X}, \mathbf{Y}$ do grupo $\Omega$.

\begin{tabular}{c|cc}
\hline$\Omega$ & $\mathrm{X}$ & $\mathrm{Y}$ \\
\hline $\mathrm{X}$ & $\mathrm{X}$ & $\mathrm{Y}$ \\
$\mathrm{Y}$ & $\mathrm{Y}$ & $\mathrm{X}$ \\
\hline
\end{tabular}

Revista Brasileira de Ensino de Física, vol. 38, nº 3, e3312, 2016 


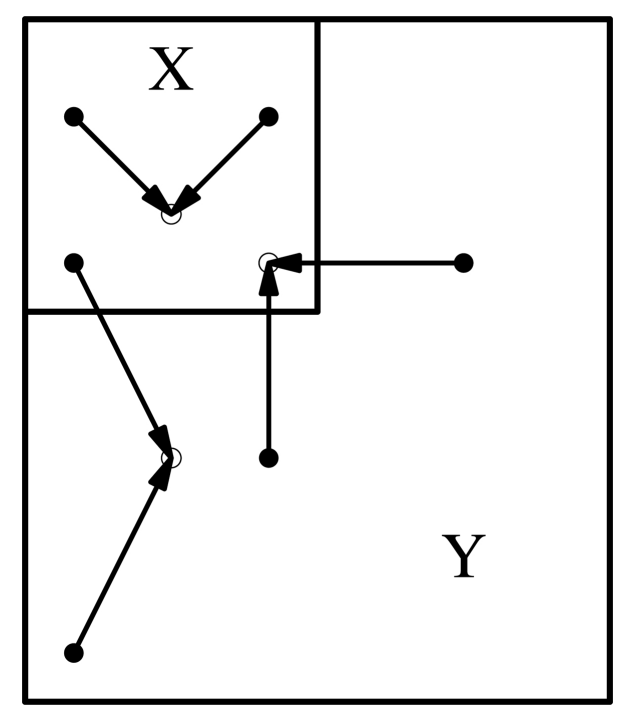

Figura 2: Esquema da estrutura do conjunto total $\Omega$.

\section{Forma Explícita de Spinores de Partícula e Antipartícula}

O estudo da estrutura algébrica do conjunto de transformações nos leva à conclusão de que todas as representações são equivalentes, isto é, uma representação pode ser obtida a partir de qualquer outra por uma transformação unitária. Usaremos este fato para a determinação da forma explícita de spinores constantes em qualquer representação a partir de um spinor de uma certa representação. Por exemplo, determinaremos a forma explícita do spinor $u_{p}^{[3]}$ de partícula na representação principal [3]: $\beta=\sigma^{3}, \alpha=\sigma^{1}$. Resolvemos a Eq. (54) para $u_{p}$. Escrevemos a equação na forma matricial

$$
\left(\begin{array}{cc}
m-\varepsilon & -p_{1} \\
-p_{1} & -m-\varepsilon
\end{array}\right)\left(\begin{array}{l}
\xi \\
\eta
\end{array}\right)=0, u_{p}=\left(\begin{array}{l}
\xi \\
\eta
\end{array}\right)
$$

que corresponde ao par das equações com respeito às componentes:

$$
\begin{aligned}
& -(\varepsilon-m) \xi-p_{1} \eta=0, \\
& -p_{1} \xi-(\varepsilon+m) \eta=0 .
\end{aligned}
$$

Podemos expressar a componente $\eta$ como:

$$
\eta=\frac{-p_{1}}{(\varepsilon+m)} \xi
$$

portanto,

$$
u_{p}=\left(\begin{array}{c}
\xi \\
\eta
\end{array}\right)=\left(\begin{array}{c}
1 \\
\frac{-p_{1}}{(\varepsilon+m)}
\end{array}\right) \xi
$$

Exigindo a normalização do spinor na forma

$$
u_{p}^{\dagger} u_{p}=1 \text {, }
$$

obtemos

$$
\begin{aligned}
& |\xi|^{2}\left(1+\frac{p_{1}^{2}}{(\varepsilon+m)^{2}}\right) \\
& =|\xi|^{2} \frac{\varepsilon^{2}+2 \varepsilon m+m^{2}+p_{1}^{2}}{(\varepsilon+m)^{2}}=|\xi|^{2} \frac{2 \varepsilon^{2}+2 \varepsilon m}{(\varepsilon+m)^{2}} \\
& =|\xi|^{2} \frac{2 \varepsilon(\varepsilon+m)}{(\varepsilon+m)^{2}} \\
& =|\xi|^{2} \frac{2 \varepsilon}{(\varepsilon+m)}=1 \Rightarrow \xi=\sqrt{\frac{\varepsilon+m}{2 \varepsilon}} e^{i \varphi},
\end{aligned}
$$

onde $\varphi$ é um fator de fase arbitrário. Portanto,

$$
\begin{aligned}
& u_{p}=\left(\begin{array}{c}
1 \\
\frac{-p_{1}}{(\varepsilon+m)}
\end{array}\right) \sqrt{\frac{\varepsilon+m}{2 \varepsilon}} e^{i \varphi} \\
& =\frac{e^{i \varphi}}{\sqrt{2 \varepsilon(\varepsilon+m)}}\left(\begin{array}{c}
\varepsilon+m \\
-p_{1}
\end{array}\right) .
\end{aligned}
$$

Impondo $\varphi=0$, obtemos:

$$
u_{p}^{[3]}=n_{f}\left(\begin{array}{c}
\varepsilon+m \\
-p_{1}
\end{array}\right), n_{f}=\frac{1}{\sqrt{2 \varepsilon(\varepsilon+m)}} .
$$

Os spinores em outras representações são obtidos a partir de $u_{p}^{[3]}$ com o uso das transformações determinadas na Seção 5:

$$
u_{p}^{\left[i_{q}\right]}=U_{i_{q} 3_{0}} u_{p}^{[3]}=U_{i_{q} i_{0}} U_{i_{0} 3_{0}} u_{p}^{[3]},
$$

onde as transformações $U_{i_{q} i_{0}}$ foram apresentadas na Tabela (6), e $U_{i_{0} 3_{0}}$ foram escritas na Eq. (84) e na Tabela (3).

Escrevendo a equação para spinores de antipartícula da Eq. (54) na forma:

$$
\left[-\alpha p_{1}-\beta m\right] v_{p}=-\varepsilon_{-} v_{p}=\varepsilon_{+} v_{p},
$$

observamos que esta equação difere da equação para spinores de partícula pelo sinal oposto do termo com $\beta$. Portanto, se é usada uma representação $\operatorname{com} \beta=s \sigma^{i}$, o spinor de antipartícula obedece à equação para o spinor de partícula na representação $\operatorname{com} \beta=-s \sigma^{i}$. De acordo com a Tabela (2) podemos estabelecer as relações entre spinores de antipartícula e spinores de partícula nas representações correspondentes:

$$
v_{p}^{\left[i_{0}\right]}=u_{p}^{\left[i_{2}\right]}, v_{p}^{\left[i_{2}\right]}=u_{p}^{\left[i_{0}\right]} ; v_{p}^{\left[i_{1}\right]}=u_{p}^{\left[i_{3}\right]}, v_{p}^{\left[i_{3}\right]}=u_{p}^{\left[i_{1}\right]} .
$$

Com o uso das transformações $U_{i_{q} i_{0}}$ da Tabela (6) escrevemos os spinores de antipartícula a partir do 
spinor de partícula da representação principal correspondente:

$$
\begin{aligned}
& v_{p}^{\left[i_{0}\right]}=u_{p}^{\left[i_{2}\right]}=U_{i_{2}, i_{0}} u_{p}^{\left[i_{0}\right]}, v_{p}^{\left[i_{2}\right]}=u_{p}^{\left[i_{0}\right]}, \\
& v_{p}^{\left[i_{1}\right]}=u_{p}^{\left[i_{3}\right]}=U_{i_{3}, i_{0}} u_{p}^{\left[i_{0}\right]}, v_{p}^{\left[i_{3}\right]}=u_{p}^{\left[i_{1}\right]} \\
& =U_{i_{1}, i_{0}} u_{p}^{\left[i_{0}\right]}
\end{aligned}
$$

No caso particular da representação [3], obtemos, como um exemplo, a forma explícita do spinor de antipartícula:

$$
\begin{gathered}
v_{p}^{[3]}=v_{p}^{\left[3_{0}\right]}=u_{p}^{\left[3_{2}\right]}=U_{3_{2}, 3_{0}} u_{p}^{\left[3_{0}\right]}=\sigma^{1} u_{p}^{[3]} \\
=n_{f} \sigma^{1}\left(\begin{array}{c}
\varepsilon+m \\
-p_{1}
\end{array}\right)=n_{f}\left(\begin{array}{c}
-p_{1} \\
\varepsilon+m
\end{array}\right) .
\end{gathered}
$$

Notamos que o caso de $2+1$ dimensões já não exibe a propriedade de equivalência de todas as representações e a possibilidade de obtenção de spinores de antipartícula a partir de spinores de partícula por uma transformação unitária.

\section{Discussão}

Nesta seção discutiremos algumas aplicações dos resultados obtidos no trabalho.

1. Nos trabalhos [23] e 24] é elaborado o método de transformadas de Darboux para a equação de Dirac em $1+1$ dimensões. O método permite gerar um hamiltoniano novo, junto com suas autofunções $\left\{\chi_{D}\right\}$, a partir de um hamiltoniano iniciante, cujo espectro e autofunções $\left\{\psi_{D}\right\}$ são conhecidos. Simbolicamente escrevemos a aplicação do método como:

$$
\chi_{D}=T D \psi_{D} .
$$

O método é elaborado para o hamiltoniano que na nossa notação é escrito como:

$$
\hat{H}_{D 0}=i \sigma^{2} \partial_{1}+v_{0}, \hat{H}_{D 0} \psi=\varepsilon \psi,
$$

onde $v_{0}$ é um potencial de interação de forma matricial. Na Eq. (163) é usada a representação de $\alpha=-\sigma^{2}$ e $\beta=\sigma^{1}$ ou $\beta=\sigma^{3}$. De acordo com a classificação proposta neste artigo, estas representações são $\left[1_{1}\right]$ ou $\left[6_{1}\right]$.

Para utilizar o método de transformadas de Darboux para um hamiltoniano $\widehat{H}_{0}$, em qualquer outra representação $\left[i_{q}\right]$, é suficiente aplicar a transformação $U_{1_{1} i_{q}}$ (ou $U_{6_{1} i_{q}}$ ) no hamiltoniano e nas suas autofunções $\{\psi\}$ para levá-los à representação usada no método:

$$
\hat{H}_{D 0}=U \hat{H}_{0} U^{\dagger}, \psi_{D}=U \psi .
$$

Em processo de aplicação do método de transformadas de Darboux são gerados o hamiltoniano novo $\widehat{H}_{D 1}$ e suas autofunções $\left\{\chi_{D}\right\}$ na representação $\left[1_{1}\right]$ (ou $\left.\left[6_{1}\right]\right)$. Se necessário, é facil voltar à representação inicial, aplicando a transformação inversa $U_{i_{q} 1_{1}}$ (ou $\left.U_{i_{q} 6_{1}}\right)$. Expressamos as etapas de cálculos simbolicamente como:

$$
\psi \rightarrow U \psi \rightarrow \psi_{D} \rightarrow T D \psi_{D} \rightarrow \chi_{D} \rightarrow U^{-1} \chi_{D} \rightarrow \chi
$$

Para as transformações diretas temos:

$$
\begin{aligned}
& U_{1_{1} i_{q}}=U_{1_{1} 1_{0}} U_{1_{0} i_{0}} U_{i_{0} i_{q}}=\sigma^{1} U_{1_{0} i_{0}} U_{i_{0} i_{q}} \\
& U_{6_{1} i_{q}}=U_{6_{1} 6_{0}} U_{6_{0} i_{0}} U_{i_{0} i_{q}}=\sigma^{3} U_{6_{0} i_{0}} U_{i_{0} i_{q}}
\end{aligned}
$$

e, para as transformações inversas,

$$
U_{i_{q} 1_{1}}=U_{i_{q} i_{0}} U_{i_{0} 1_{0}} \sigma^{1} ; U_{i_{q} 6_{1}}=U_{i_{q} i_{0}} U_{i_{0} 6_{0}} \sigma^{3} .
$$

2. Discutiremos resumidamente também a existência de férmions de Majorana em $1+1$ dimensões e sua forma explícita. Segundo as Refs. 25 e [26], os férmions de Majorana podem ser definidos como as soluções da equação de Dirac, em que é usada a representação em que as componentes das matrizes $\gamma$ são imaginárias. No caso de $1+1$ dimensões estas representações são $\left[2_{q}\right]$ e $\left[5_{q}\right]$. Um spinor de Majorana obedece a condição

$$
\psi=\gamma^{0} C \psi^{*},
$$

onde o simbolo * é a conjugação complexa, $C$ é uma matriz unitária que obedece a condição:

$$
C^{\top}=-C
$$

o simbolo ${ }^{\top}$ indica a matriz transposta. Os spinores constantes de partícula e antipartícula de um férmion de Majorana obedecem às condições:

$$
v_{p}=\gamma^{0} C u_{p}^{*}, u_{p}=\gamma^{0} C v_{p}^{*} .
$$

Determinaremos a forma explícita da matriz $C$. Escrevendo a matriz $C$ na base $\left\{\sigma^{0}, \sigma^{i}\right\}$

$$
C=c_{\mu} \sigma^{\mu}=c_{0} \sigma^{0}+c_{1} \sigma^{1}+c_{2} \sigma^{2}+c_{3} \sigma^{3}
$$

e usando as relações das matrizes de Pauli

$$
\begin{aligned}
& \left(\sigma^{0}\right)^{\top}=\sigma^{0},\left(\sigma^{1}\right)^{\top}=\sigma^{1}, \\
& \left(\sigma^{2}\right)^{\top}=-\sigma^{2},\left(\sigma^{3}\right)^{\top}=\sigma^{3},
\end{aligned}
$$

Revista Brasileira de Ensino de Física, vol. 38, nº 3, e3312, 2016 
temos:

$$
C^{\top}=c_{0} \sigma^{0}+c_{1} \sigma^{1}-c_{2} \sigma^{2}+c_{3} \sigma^{3} .
$$

A condição 164 leva à relação

$$
\begin{aligned}
& c_{0} \sigma^{0}+c_{1} \sigma^{1}-c_{2} \sigma^{2}+c_{3} \sigma^{3} \\
& =-c_{0} \sigma^{0}-c_{1} \sigma^{1}-c_{2} \sigma^{2}-c_{3} \sigma^{3},
\end{aligned}
$$

que dá

$$
c_{0}=c_{1}=c_{3}=0 \Rightarrow\left|c_{2}\right|^{2}=1 \Rightarrow c_{2}=e^{i \varphi} .
$$

Portanto

$$
C=e^{i \varphi} \sigma^{2}
$$

onde $\varphi$ é um fator de fase arbitrário.

Determinaremos a forma explícita de spinores constantes de partícula e antipartícula de férmions de Majorana para as representações principais [2] e [5]. Na representação [2] eles são:

$$
\begin{gathered}
u_{p}^{[2]}=U_{23} u_{p}^{[3]}=\frac{n_{f}}{\sqrt{2}}\left(\begin{array}{cc}
1 & 1 \\
i & -i
\end{array}\right)\left(\begin{array}{c}
\varepsilon+m \\
-p_{1}
\end{array}\right)=\frac{n_{f}}{\sqrt{2}}\left(\begin{array}{c}
\varepsilon+m-p_{1} \\
i\left(\varepsilon+m+p_{1}\right.
\end{array}\right), \\
v_{p}^{[2]}=u_{p}^{\left[2_{2}\right]}=U_{2_{2}, 2_{0}} u_{p}^{\left[2_{0}\right]}=\frac{n_{f}}{\sqrt{2}} \sigma^{3}\left(\begin{array}{c}
\varepsilon+m-p_{1} \\
i\left(\varepsilon+m+p_{1}\right)
\end{array}\right)=\frac{n_{f}}{\sqrt{2}}\left(\begin{array}{c}
\varepsilon+m-p_{1} \\
-i\left(\varepsilon+m+p_{1}\right)
\end{array}\right) .
\end{gathered}
$$

Na representação [5] eles são:

$$
\begin{gathered}
u_{p}^{[5]}=U_{53} u_{p}^{[3]}=\frac{n_{f}}{\sqrt{2}}\left(\begin{array}{cc}
1 & i \\
i & 1
\end{array}\right)\left(\begin{array}{c}
\varepsilon+m \\
-p_{1}
\end{array}\right)=\frac{n_{f}}{\sqrt{2}}\left(\begin{array}{c}
\varepsilon+m-i p_{1} \\
i(\varepsilon+m)-p_{1}
\end{array}\right), \\
v_{p}^{[5]}=u_{p}^{\left[5_{2}\right]}=U_{5_{2}, 5_{0}} u_{p}^{\left[5_{0}\right]}=\frac{n_{f}}{\sqrt{2}} \sigma^{1}\left(\begin{array}{c}
\varepsilon+m-i p_{1} \\
i(\varepsilon+m)-p_{1}
\end{array}\right)=\frac{n_{f}}{\sqrt{2}}\left(\begin{array}{c}
i(\varepsilon+m)-p_{1} \\
\varepsilon+m-i p_{1}
\end{array}\right) .
\end{gathered}
$$

Justificaremos as relações 165 para as representações [2] e [5]. Para a representação [2] usamos $C=\sigma^{2}$, que dá $\gamma^{0} C=\sigma^{2} \sigma^{2}=1$, portanto

$$
\gamma^{0} C u_{p}^{[2] *}=\frac{n_{f}}{\sqrt{2}}\left(\begin{array}{c}
\varepsilon+m-p_{1} \\
-i\left(\varepsilon+m+p_{1}\right)
\end{array}\right)=v_{p}^{[2]}
$$

Para representação [5] usamos $C=i \sigma^{2}$, que dá $\gamma^{0} C=\sigma^{2} i \sigma^{2}=i$, portanto

$$
\gamma^{0} C u_{p}^{[5] *}=\frac{i n_{f}}{\sqrt{2}}\left(\begin{array}{c}
\varepsilon+m+i p_{1} \\
-i(\varepsilon+m)-p_{1}
\end{array}\right)=\frac{n_{f}}{\sqrt{2}}\left(\begin{array}{c}
i(\varepsilon+m)-p_{1} \\
(\varepsilon+m)-i p_{1}
\end{array}\right)=v_{p}^{[5]}
$$

\section{Conclusão}

Neste trabalho consideramos as representações da equação de Dirac em $1+1$ dimensões. Introduzimos uma classificação unificada de todas as representações nas quais cada matriz de Dirac é associada a uma única matriz de Pauli. Esta classificação inclui 6 representações principais: 3 cíclicas e 3 anticíclicas. A inclusão das representações com sinais modificados das matrizes de Pauli faz com que cada representação principal torne-se um membro de um quarteto das representações. O número total das representações distintas portanto é 24. Determinamos todas as transformações unitárias entre as representações na forma explícita. Formalmente, o número das transformações é 552, mas grande parte delas é expressa na mesma forma. As transformações entre as representações cíclicas (anticíclicas) principais são expressas por duas transformações diferentes. As transformações entre representações cíclicas e anticíclicas são expressas por 9 transformações diferentes. Entre elas, 3 são hermitiano conjugadas e outras 6 compõem 3 pares em que um membro do par é o resultado da conjugação hermitiana do outro. As transformações entre os membros de todos os quartetos das representações são expressas por três matrizes de Pauli. Estudamos a estrutura algébrica do conjunto das transformações. Estabelecemos que o conjunto das transformações forma um grupo não abeliano com respeito ao produto matri- 
cial em classes de equivalência, definidas através da indistinguibilidade das transformações que diferem por um fator de fase constante. Este grupo possui um subgrupo não trivial, o qual por sua vez, contém os dois subgrupos não triviais. O estudo de estrutura do conjunto leva à conclusão de que todas as representações são equivalentes, isto é, uma representação pode ser obtida a partir de qualquer outra por uma transformação unitária. A forma explícita de spinores constantes em qualquer representação pode ser determinada a partir de um spinor de uma certa representação por uma transformação unitária. Além disso, spinores constantes de antipartícula podem ser determinados, a partir de spinores de partícula, por uma transformação unitária apropriada no caso livre. Notamos que o caso de $2+1$ dimensões já não exibe estas propriedades.

Como aplicação dos resultados obtidos no trabalho, apresentamos uma adição útil ao método de transformadas de Darboux para a equação de Dirac em $1+1$ dimensões. Discutimos resumidamente a existência de férmions de Majorana em 1+1 dimensões e apresentamos exemplos da sua forma explícita. Apresentamos também a forma geral das matrizes de Dirac em $1+1$ dimensões determinada por 3 parâmetros arbitrários. A forma geral pode ser útil em discussões de problemas em dimensões mais altas que $1+1$, por exemplo, no caso de uso de coordenadas especiais ou para interpretações de resultados. Demonstramos que há um isomorfismo entre o par das matrizes de Dirac e o par de vetores unitários ortogonais, ou por outro ponto de vista, isomorfismo entre o par das matrizes de Dirac e um intervalo geodésico direcionado de comprimento $\pi / 2$ na variedade $S^{2}$. Em virtude disto, indicamos que a tarefa de classificação de representações e a busca de transformações unitárias entre elas pode ser convertida numa formulação geométrica.

Como uma continuação deste trabalho vemos uma consideração de aspectos análogos da equação de Dirac em $2+1$ dimensões.

\section{Apêndice}

No caso geral, as matrizes de Dirac $\beta, \alpha$ são matrizes complexas $2 \times 2$ que obedecem às seguintes condições. Elas são hermitianas

$$
\beta^{\dagger}=\beta, \alpha^{\dagger}=\alpha
$$

com traço nulo

$$
\operatorname{Tr}(\beta)=\operatorname{Tr}(\alpha)=0,
$$

seu quadrado é igual à unidade

$$
\alpha^{2}=\beta^{2}=1
$$

e elas anticomutam

$$
\{\alpha, \beta\}=\alpha \beta+\beta \alpha=0 .
$$

Escrevemos $\beta, \alpha$ na base de matrizes de Pauli $\sigma^{i}$ e a matriz unidade $\sigma^{0}$ :

$$
\begin{aligned}
& \beta=b_{\mu} \sigma^{\mu}=b_{0} \sigma^{0}+b_{i} \sigma^{i}=\left(\begin{array}{cc}
b_{0}+b_{3} & b_{1}-i b_{2} \\
b_{1}+i b_{2} & b_{0}-b_{3}
\end{array}\right), b_{\mu} \in \mathbb{C}, \\
& \alpha=a_{\mu} \sigma^{\mu}=a_{0} \sigma^{0}+a_{i} \sigma^{i}=\left(\begin{array}{cc}
a_{0}+a_{3} & a_{1}-i a_{2} \\
a_{1}+i a_{2} & a_{0}-a_{3}
\end{array}\right), a_{\mu} \in \mathbb{C} .
\end{aligned}
$$

As matrizes são definidas por coeficientes $b_{\mu}, a_{\mu}$ os quais agrupamos do seguinte modo:

$$
\beta=\left(b_{0}, b_{i}\right)=\left(b_{0}, \vec{b}\right), \alpha=\left(a_{0}, a_{i}\right)=\left(a_{0}, \vec{a}\right), b_{\mu}, a_{\mu} \in \mathbb{C} .
$$

Com o uso desta notação podemos escrever o resultado do produto das matrizes como:

$$
\alpha \beta=\left(a_{0} b_{0}+a_{i} b_{i}, a_{0} b_{i}+b_{0} a_{i}+i \varepsilon_{i j k} a_{j} b_{k}\right)=\left(a_{0} b_{0}+\vec{a} \vec{b}, a_{0} \vec{b}+b_{0} \vec{a}+i \vec{a} \times \vec{b}\right)
$$

e o quadrado das matrizes como:

$$
\alpha^{2}=\left(a_{0}^{2}+\vec{a}^{2}, 2 a_{0} \vec{a}+i \vec{a} \times \vec{a}\right)=\left(a_{0}^{2}+\vec{a}^{2}, 2 a_{0} \vec{a}\right),
$$




$$
\beta^{2}=\left(b_{0}^{2}+\vec{b}^{2}, 2 b_{0} \vec{b}\right) .
$$

As matrizes hermitiano-conjugadas são dadas por:

$$
\beta^{\dagger}=b_{\mu}^{*} \sigma^{\mu}, \alpha^{\dagger}=a_{\mu}^{*} \sigma^{\mu},
$$

onde o símbolo * denota conjugação complexa.

A condição de hermiticidade de $\beta, \alpha$ leva às relações:

$$
b_{\mu}^{*} \sigma^{\mu}=b_{\mu} \sigma^{\mu}, a_{\mu}^{*} \sigma^{\mu}=a_{\mu} \sigma^{\mu} \Rightarrow b_{\mu}^{*}=b_{\mu}, a_{\mu}^{*}=a_{\mu} \Rightarrow b_{\mu}, a_{\mu} \in \mathbb{R} .
$$

A condição de traço nulo implica:

$$
\operatorname{Tr}(\beta)=2 b_{0}=0, \operatorname{Tr}(\alpha)=2 a_{0}=0 \Rightarrow b_{0}=0, a_{0}=0 .
$$

Com o uso das últimas relações para o quadrado das matrizes temos:

$$
\beta^{2}=\left(\vec{b}^{2}, 0\right)=1, \alpha^{2}=\left(\vec{a}^{2}, 0\right)=1 \Rightarrow \vec{b}^{2}=1, \vec{a}^{2}=1 .
$$

Finalmente, a condição de anticomutação das matrizes implica em:

$$
\{\alpha, \beta\}=\alpha \beta+\beta \alpha=(\vec{a} \vec{b}, i \vec{a} \times \vec{b})+(\vec{b} \vec{a}, i \vec{b} \times \vec{a})=(2 \vec{a} \vec{b}, 0)=0 \Rightarrow \vec{a} \vec{b}=0 .
$$

Portanto, podemos concluir que as matrizes de Dirac $\beta, \alpha$ são isomorfas ao par de vetores unitários ortogonais com seu início na origem:

$$
\beta \sim \vec{b}, \alpha \sim \vec{a} ;|\vec{b}|=|\vec{a}|=1, \vec{a} \vec{b}=0 .
$$

As componentes dos vetores $\vec{b}, \vec{a}$ definem os coeficientes das matrizes $\beta, \alpha$ na decomposição pela base $\left\{\sigma^{0}, \sigma^{i}\right\}$ :

$$
\begin{aligned}
& \beta=b_{i} \sigma^{i}=\left(\begin{array}{cc}
b_{3} & b_{1}-i b_{2} \\
b_{1}+i b_{2} & -b_{3}
\end{array}\right), b_{i} \in \mathbb{R}, \\
& \alpha=a_{i} \sigma^{i}=\left(\begin{array}{cc}
a_{3} & a_{1}-i a_{2} \\
a_{1}+i a_{2} & -a_{3}
\end{array}\right), a_{i} \in \mathbb{R} .
\end{aligned}
$$

Por exemplo, os componentes do vetor $\vec{b}$ podem ser escolhidos na forma:

$$
b_{i}=\sin \theta \cos \varphi, b_{j}=\sin \theta \sin \varphi, b_{k}=\cos \theta
$$

com os dois parâmetros arbitrários $\theta, \varphi$. As componentes do vetor $\vec{a}$ que é localizado no plano perpendicular ao vetor $\vec{b}$ são dadas por:

$$
a_{i}=\cos \theta \cos \varphi \cos \chi-\sin \varphi \sin \chi, a_{j}=\cos \theta \sin \varphi \cos \chi+\cos \varphi \sin \chi, a_{k}=-\sin \theta \cos \chi
$$

com um parâmetro arbitrário $\chi$. Para as matrizes $\beta, \alpha$ temos:

$$
\begin{gathered}
\beta=\sigma^{1} \sin \theta \cos \varphi+\sigma^{2} \sin \theta \sin \varphi+\sigma^{3} \cos \theta=\left(\begin{array}{cc}
\cos \theta & e^{-i \varphi} \sin \theta \\
e^{i \varphi} \sin \theta & -\cos \theta
\end{array}\right) \\
\alpha=\sigma^{1}(\cos \theta \cos \varphi \cos \chi-\sin \varphi \sin \chi)+\sigma^{2}(\cos \theta \sin \varphi \cos \chi+\cos \varphi \sin \chi)+\sigma^{3}(-\sin \theta \cos \chi) \\
=\left(\begin{array}{cc}
-\sin \theta \cos \chi & e^{-i \varphi}(\cos \theta \cos \chi-i \sin \chi) \\
e^{i \varphi}(\cos \theta \cos \chi+i \sin \chi) & \sin \theta \cos \chi
\end{array}\right) .
\end{gathered}
$$

No caso particular $\chi=0$ temos:

$$
a_{i}=\cos \theta \cos \varphi, a_{j}=\cos \theta \sin \varphi, a_{k}=-\sin \theta
$$

e

$$
\alpha=\sigma^{1} \cos \theta \cos \varphi+\sigma^{2} \cos \theta \sin \varphi+\sigma^{3}(-\sin \theta)=\left(\begin{array}{cc}
-\sin \theta & e^{-i \varphi} \cos \theta \\
e^{i \varphi} \cos \theta & \sin \theta
\end{array}\right) .
$$


A Eq. (166) pode ser interpretada de outra maneira. As matrizes de Dirac $\beta, \alpha$ são isomorfas aos pontos na superfície de uma esfera. Os pontos indicam o início e o fim de um intervalo curvado de comprimento $\pi / 2$ na superfície de uma esfera de raio igual a unidade. O par das matrizes de Dirac $\beta, \alpha$ é isomorfo a um intervalo geodésico direcionado de comprimento $\pi / 2$ na variedade $S^{2}$. Então, a tarefa de classificação das representações e a busca de transformações unitárias entre elas, pode ser reformulada geometricamente.

\section{Agradecimentos}

Agradecemos à Fundação de Apoio à Pesquisa e à Inovação Tecnológica do Estado de Sergipe (FAPITEC-SE) pelo apoio financeiro parcial deste trabalho. Gostariamos também de agaredecer ao Prof. Osmar de Souza e Silva Júnior, ao Prof. Gerson Cortês Duarte Filho e Thainá Aragão Sabino do Departamento de Física da Universidade Federal de Sergipe por suas contribuições na revisão final do texo do artigo.

\section{Referências}

[1] L.I. Schiff, Quantum Mechanics (McGraw-Hill, New York, 1968).

[2] W. Greiner, Relativistic Quantum Mechanics (Springer, Berlin, 2000).

[3] J.D. Bjorken and S.D. Drell, Relativistic Quantum Mechanics (McGraw-Hill, New York, 1964).

[4] F. Mandl and G. Shaw, Quantum Field Theory (John Wiley, Chichester, 1984).

[5] L.H. Ryder, Quantum Field Theory (Cambridge University Press, Cambridge, 1996).
[6] C. Itzykson and J.-B. Zuber, Quantum Field Theory (McGraw-Hill, New York, 1980).

[7] M.E. Peskin and D.V. Schroeder, An introduction to Quantum Field Theory (Addison-Wesley, Boston, 1995).

[8] B. Thaller, The Dirac Equation (Springer-Verlag, Berlin, 1992).

[9] V.G. Bagrov and D.M. Gitman, The Dirac Equation and its Solutions (Walter de Gruyter, Berlin, 2014).

[10] K. Fredenhagen, in: Fields and Particles (SpringerVerlag, Berlin, 1990), p. 53-87.

[11] H. Galic, Am. J. Phys. 56, 312 (1988).

[12] J.R. Hiller, Am. J. Phys. 70, 522 (2002).

[13] R.S. Bhalerao and B. Ram, Am. J. Phys. 69, 817 (2001).

[14] Y. Nogami, F.M. Toyama and W. van Dijk, Am. J. Phys. 71, 950 (2003).

[15] V. Alonsoy and S. De Vincenzoz, J. Phys. A 30, 8573 (1997)

[16] O. Mustafa, Cent. Eur. J. Phys. 11, 480 (2013).

[17] L.A. González-Díaz, A.A. Díaz, S. Díaz-Solórzano and J.R. Darias, J. Phys. A 48, 045207 (2015).

[18] Jian-Yuan Cheng, Found. Phys. 44, 953 (2014).

[19] J. Bunao and Eric A. Galapon, Ann. Phys. 356, 369 (2015).

[20] Luiz P. de Oliveira and Luis B. Castro, Ann. Phys. 365, 99 (2016).

[21] G.B. Arfken and H.J. Weber, Física Matemática. Métodos Matemáticos para Engenharia e Física (Campus Elsevier, São Paulo, 2005).

[22] A.C. Muniz Neto, Tópicos de Matemática Elementar (SBM, Rio de Janeiro, 2012).

[23] N. Debergh, A.A. Pecheritsin, B.F. Samsonov and B. Van den Bossche, J. Phys. A 35, 3279 (2002).

[24] L.M. Nieto, A.A. Pecheritsin and B.F. Samsonov, Ann. Phys. 305, 151 (2003).

[25] P.B. Pal, arXiv:physics/0703214.

[26] P.B. Pal, Am. J. Phys. 79, 485 (2011). 